ESAIM: PROCEEDINGS, December 2013, Vol. 42, p. 34-60

A. Heibig, F. Filbet, L. I. Palade, Editors

\title{
APPROXIMATING THE TRACE OF ITERATIVE SOLUTIONS AT THE INTERFACES WITH NONUNIFORM FOURIER TRANSFORM AND SINGULAR VALUE DECOMPOSITION FOR COST-EFFECTIVELY ACCELERATING THE CONVERGENCE OF SCHWARZ DOMAIN DECOMPOSITION
}

\author{
Damien Tromeur-Dervout ${ }^{1}$
}

\begin{abstract}
Résumé. Cet acte traite de la représentation des solutions itérées aux interfaces de la méthode de décomposition de domaine de type Schwarz afin d'approximer de manière adaptative son opérateur d'erreur aux interfaces des sous domaines. Ceci permet de construire de manière économique l'accélération de la convergence de la méthode itérative en étendant la technique d'accélération de la convergence de Aitken au cas vectoriel. La première représentation est fondée sur la construction d'une transformée de Fourier discrète non uniforme définie sur un maillage non régulier. Nous montrons comment construire une base de Fourier de dimension $\mathrm{N}+1$ sur ce maillage à partir de la construction numérique d'une forme sesquilinéaire, son exactitude pour les polynômes trigonométriques de degré $\mathrm{N} / 2$, et numériquement sa capacité d'approximation spectrale dépendante de la continuité de la fonction à approximer. La décroissance des modes de Fourier de la trace de la solution itérée aux interfaces nous fournis une estimation pour sélectionner de manière adaptive les modes intervenant dans l'accélération. Le défaut de cette approche est d'être dépendant de la continuité de la solution itérée aux interfaces. La deuxième représentation, purement algébrique, utilise une décomposition en valeurs singulières des solutions itérées aux interfaces pour fournir un ensemble de vecteurs singuliers orthogonaux dont les valeurs singulières associées fournissent une estimation pour adapter l'accélération. La méthode Aitken-Schwarz résultante est ensuite appliquée au calcul à grande échelle pour résoudre un écoulement 3D en milieu poreux modélisé par l'équation de Darcy linéaire où la perméabilité suit une distribution aléatoire log-normale.
\end{abstract}

${ }^{1}$ University of Lyon, University Lyon1 CNRS Institut Camille Jordan UMR5208, 43 Bd du 11 Novembre 1918, F-69150 Villeurbanne cedex, France

(C) EDP Sciences, SMAI 2013 


\begin{abstract}
This paper deals with the representation of the trace of iterative Schwarz solutions at the interfaces of domain decomposition to approximate adaptively the interface error operator. This allows to build a cost-effectively accelerating of the convergence of the iterative method by extending to the vectorial case the Aitken's accelerating convergence technique. The first representation is based on the building of a nonuniform discrete Fourier transform defined on a non-regular grid. We show how to construct a Fourier basis of dimension $\mathrm{N}+1$ on this grid by building numerically a sesquilinear form, its exact accuracy to represent trigonometric polynomials of degree $\mathrm{N} / 2$, and its spectral approximation property that depends on the continuity of the function to approximate. The decay of Fourier-like modes of the approximation of the trace of the iterative solution at the interfaces provides an estimate to adaptively select the modes involved in the acceleration. The drawback of this approach is to be dependent on the continuity of the trace of the iterated solution at the interfaces. The second representation, purely algebraic, uses a singular value decomposition of the trace of the iterative solution at the interfaces to provide a set of orthogonal singular vectors of which the associated singular values provide an estimate to adapt the acceleration. The resulting Aitken-Schwarz methodology is then applied to large scale computing on 3D linear Darcy flow where the permeability follows a log normal random distribution.
\end{abstract}

\title{
INTRODUCTION
}

The Schwarz domain decomposition (DD) method[35] is a very attractive numerical method for the parallel computing as it needs only to update the boundary conditions on the artificial interfaces generated by DD. Thus only local communications between the neighboring sub-domains are needed. Nevertheless, the main drawback of this method is its slow rate of convergence which depends on the partial differential problem, the geometry of the sub-domains, and the size of the overlap when overlap is present. The idea of using Aitken acceleration [20] on the classical additive Schwarz DD method was introduced in [18]. These authors have called the corresponding method the Aitken-Schwarz (AS) method. They have shown its great numerical performances on linear and nonlinear elliptic problems discretized by a five-point scheme on a rectangular Cartesian grid [2]. It was shown that this technique gives efficient metacomputing time to solve elliptic linear separable problem with the Laplacian operator and nonlinear problem such as the Bratu problem [5] in three dimensional space with a broad network of supercomputers linked by a regular internet connection.

For an elliptic separable operator with constant coefficients on a mesh with regular step sizes, the AS method is a direct solver. One can use Fourier transform of the trace of the Schwarz iterative solution at the interfaces and the Aitken acceleration of convergence formula can be applied on each Fourier mode in the Fourier space. When the operators are no more separable, the Aitken acceleration involves an operator that couples the Fourier modes. Then the Aitken acceleration cannot be scalar and the acceleration is vectorial and involves a full matrix of size the number of unknowns on all the DD interfaces. Consequently some approximation of the Aitken acceleration operator is required to save computing cost. As this DD purely linear convergence behaviour depends on the error operator at the artificial interfaces, the proposed methodology consists to approximate the trace of the iterative DD solution at the interfaces in a set of vectors orthogonal with respect to a scalar product or a sesquilinear form. The efficiency of the acceleration depends on the property of the set of vectors to represent the searched solution. Moreover, in order to reduce the size of the error operator, the approximation should have a decay property of its coefficients in the basis. In order to focus on these points, we illustrate the Aitken-Schwarz methodology with two representations of the trace of the iterative solution on the DD interfaces. The first representation is based on the building of a non-uniform discrete Fourier transform defined on a nonregular grid. The accuracy of this approximation is related to the continuity of the solution to approximate. The second representation, purely algebraic, uses a singular value decomposition of the trace of the iterative solution at the interfaces to provide a set of orthogonal singular vectors. The interest of this paper with two approximation methods exposed is to understand that are the same mechanisms of orthogonality in the vector 
basis and decay in the coefficients of the approximation that provide an estimate to adapt the vectorial Aitken acceleration technique of the Schwarz DD.

The plan of the paper is the following. In section 1 we present the Schwarz DD method and its convergence property when applied to linear elliptic problems. Then section 2 focuses on the Aitken's acceleration of the convergence of Schwarz DD method for linear separable operators on regular meshes. Difficulties arise when linear non separable operators and/or with non regular mesh on the interfaces are under consideration. Section 3 defines the nonuniform Fourier-like transforms and the corresponding approximation of the error operator on the interface [14]. Section 4 extends the methodology with an Aitken acceleration based on the singular value decomposition of the solution at the artificial boundaries. Then this method becomes totally mesh free [39]. Moreover, the decreasing of the singular values gives an a priori criterion to select the singular vectors involved in the Aitken's acceleration operator approximation. This allows solving the large 3D computation of the linear Darcy equation where the permeability field follows a random log normal distribution law [3] in section 5.

\section{The SchWARZ DOMAIN DECOMPOSITION METHOD AND ITS PROPERTIES}

In the Schwarz DD method the problem is reduced to solve a problem on the interface. The trace theorem (see for example [[37],p.9] and references) allows to define a restriction of the solution on the interface and also to extend the interface solution in the whole subdomains. Let $\Omega \subset \mathbb{R}^{n}$ be a bounded domain with Lipschitz boundary $\Gamma:=\partial \Omega$. For any $u$ belonging to the Hilbert space $H^{1}(\Omega)$ there exists the trace $\gamma_{0} u \in H^{1 / 2}(\Gamma)$ satisfying

$$
\left\|\gamma_{0} u\right\|_{H^{1 / 2}(\Gamma)} \leq c_{T} \cdot\|u\|_{H^{1}(\Omega)}
$$

Vice-versa, for any $u \in H^{1 / 2}(\Gamma)$ there exists a bounded extension $\varepsilon u \in H^{1}(\Omega)$ satisfying $\gamma_{0} \varepsilon u=u$ and

$$
\|\varepsilon u\|_{H^{1}(\Omega)} \leq c_{I T} \cdot\|u\|_{H^{1 / 2}(\Gamma)} .
$$

Consider a scalar second order uniformly elliptic boundary value problem with Dirichlet boundary condition $(\mathrm{BC})$ :

$$
\begin{cases}L(x) u(x) & =f(x), \text { for } x \in \Omega \\ \gamma_{0} u(x) & =g(x), \text { for } x \in \Gamma\end{cases}
$$

where the partial differential operator $L(x), x \in \Omega$ is given by

$$
L(x) u(x)=-\sum_{i, j=1}^{n} \frac{\partial}{\partial x_{j}}\left[a_{j i}(x) \frac{\partial}{\partial x_{i}} u(x)\right]
$$

with $a_{j i}=a_{i j} \in L_{\infty}(\Omega), i, j=1, \ldots, n . L($.$) is assumed to be uniformly elliptic, i.e. there exists a positive$ constant $c_{0}$ independent of $x \in \Omega$ such that for all $x \in \Omega$

$$
\sum_{i, j=1}^{n} a_{j i}(x) \xi_{j} \xi_{l} \geq c_{0} \cdot|\xi|^{2}, \forall \xi \in \mathbb{R}^{n}
$$

We assume $f \in H^{-1}(\Omega), g \in H^{1 / 2}(\Gamma)$.

The conormal derivative operator $\gamma_{1}$ is given by

$$
\gamma_{1} u(x):=\sum_{i, j=1}^{n} n_{j}(x)\left[a_{j i}(x) \frac{\partial}{\partial x_{i}} u(x)\right], \forall x \in \Gamma
$$

where $n(x)$ is the exterior unit normal vector define almost everywhere for $x \in \Gamma$. 
For $u, v \in H^{1}(\Omega)$, we define the symmetric bilinear form

$$
a(u, v):=\Sigma_{i, j=1}^{n} \int_{\Omega} \frac{\partial}{\partial x_{j}} v(x) a_{j i}(x) \frac{\partial}{\partial x_{i}} u(x) d x
$$

which is bounded in $H^{1}(\Omega)$

$$
|a(u, v)|=c .\|u\|_{H^{1}(\Omega)}\|v\|_{H^{1}(\Omega)}, \forall u, v \in H^{1}(\Omega) .
$$

Using the Green's second formula for $u, v \in H^{1}(\Omega)$,

$$
a(u, v)=\int_{\Omega} L(x) u(x) v(x) d x+\int_{\Gamma} \gamma_{1} u(x) \gamma_{0} v(x) d s_{x}
$$

Let $H_{0}^{1}(\Omega):=\left\{v \in H^{1}(\Omega): \gamma_{0} v(x)=0\right.$, for $\left.x \in \Gamma\right\}$. Using (9), the variational formulation of Eq (3) is : Find $u \in H^{1}(\Omega)$ satisfying $\gamma_{0} u(x)=g(x), \forall x \in \Gamma$ such that

$$
a(u, v)=\int_{\Omega} f(x) v(x) d x, \forall v \in H_{0}^{1}(\Omega)
$$

From the uniform ellipticity (5) and Poincaré inequality, we get that the bilinear form $a(.,$.$) is elliptic on$ $H_{0}^{1}(\Omega)$. Using Eq (8),Eq (5), and the generalized Lax-Milgram lemma due to Necas [28], we conclude that problem (3) has a unique solution $u \in H^{1}(\Omega)$.

\subsection{The Dirichlet-Neumann Map}

The Dirichlet-Neuman map connects the solution and its normal derivative at the domain boundary. The DD method can be formulated in terms of this Dirichlet to Neumann map. The unique solution of problem (3) $u \in H^{1}(\Omega)$ can be written as $u=u_{0}+\varepsilon g$, where $u_{0}$ corresponds to the solution of Eq (3) with homogeneous Dirichlet BC and $\varepsilon g$ satisfies the homogenous Eq (3) with non-homogeneous Dirichlet BC . We define a linear functional

$$
l(w):=a(u, \varepsilon w)-\int_{\Omega} f(x) \varepsilon w(x) d x, \text { for } w \in H^{1 / 2}(\Gamma) .
$$

Applying the Riez representation theorem, there exists a $\lambda \in H^{-1 / 2}(\Gamma)$ such that

$$
\langle\lambda, w\rangle_{L_{2}(\Gamma)}=l(w), \forall w \in H^{1 / 2}(\Gamma)
$$

Hence, $\lambda \in H^{-1 / 2}(\Gamma)$ is the conormal derivative $\gamma_{1} u$, as it satisfies:

$$
\int_{\Gamma} \lambda(x) w(x) d s_{x}=a\left(u_{0}+\varepsilon g, \varepsilon w\right)-\int_{\Omega} f(x) \varepsilon w(x) d x, \text { for all } w \in H^{1 / 2}(\Gamma) .
$$

We then have defined a map from the given data $(f, g)$ to the associated Neumann boundary data $\lambda:=\gamma_{1} u$. In particular, for a fixed $f$ and varying Dirichlet boundary data $g=\gamma_{0} u$, we have defined a Dirichlet-Neumann map which may be written as

$$
\gamma_{1} u(x)=S g(x)-N f(x), \forall x \in \Gamma
$$

where $S$ is the Steklov-Poincaré operator associated to the homogeneous problem with Dirichlet BC and $N f$ is some Newton potential associated to the non-homogeneous Eq (3) with homogeneous BC . 


\subsection{The Generalized Schwarz Alternating Method}

Let us first recall the Generalized Schwarz Alternating Method (GSAM) introduced by [12] that gathers several Schwarz techniques [32]. For the sake of simplicity, let us consider the case where the whole domain $\Omega$ is decomposed into two sub-domains $\Omega_{1}$ and $\Omega_{2}$, with overlapping or not, defining two artificial boundaries $\Gamma_{1}, \Gamma_{2}$. Let us define $\bar{i}=\bmod (i, 2)+1$ and $\Omega_{11}=\Omega_{1} \backslash \Omega_{2}, \Omega_{22}=\Omega_{2} \backslash \Omega_{1}, \Omega_{12}=\Omega_{1} \cap \Omega_{2}, \Omega_{11}=\Omega_{1} \backslash \Omega_{12}$, $\Omega_{22}=\Omega_{2} \backslash \Omega_{12}$ if there is an overlap. Let $L(x)$ be the continuous elliptic operator. The GSAM solves until convergence the problem (3) restricted to the domain $\Omega_{1}$ (respectively $\Omega_{2}$ ) with the Dirichlet B.C on $\partial \Omega_{1} \Gamma_{1}$ (respectively $\partial \Omega_{2} \Gamma_{2}$ ) and a generalized BC: $\Lambda_{1} u_{1}+\lambda_{1} \frac{\partial u_{1}}{n_{1}}=\mu_{2}$ on $\Gamma_{1}$ (respectively $\Lambda_{2} u_{2}+\lambda_{2} \frac{\partial u_{2}}{n_{2}}=\mu_{1}$ ), where $\Lambda_{i}$ are some operators and $\lambda_{i}$ are constants. The value $\mu_{2}=\Lambda_{1} u_{2}+\lambda_{1} \frac{\partial u_{2}}{\partial n_{1}}$ (respectively $\mu_{1}=\Lambda_{2} u_{1}+\lambda_{2} \frac{\partial u_{1}}{\partial n_{2}}$ ) is defined with respect to the solution $u_{2}$ in $\Omega_{2}$ (respectively $u_{1}$ in $\Omega_{1}$ ). As the solution on sub-domains $\Omega_{i}$ are unknowns, we iterate the solving, taking the generalized $\mathrm{BC}$ value $\mu_{i}$ with the solution obtained at the previous iterate in $\Omega_{i}$. The additive version of the GSAM consists to solve both sub-domains problems at the same iterate while the multiplicative version the sub-domains problems are solved successively as updating the $\mu_{i}$ values. Then GSAM can be summarized in Algorithm 1 that follows:

Algorithm 1 GSAM: Multiplicative $\left(M_{1}=N_{2}=2 n+1, M_{2}=2 n+2, N_{1}=2 n\right)$, Additive $\left(M_{1}=M_{2}=n+1\right.$ $\left.N_{1}=N_{2}=n\right)$

1: DO until convergence

2: Solve

$$
\begin{aligned}
L(x) u_{1}^{M_{1}}(x) & =f(x), \forall x \in \Omega_{1} \\
u_{1}^{M_{1}}(x) & =g(x), \forall x \in \partial \Omega_{1} \backslash \Gamma_{1}, \\
\Lambda_{1} u_{1}^{M_{1}} & +\lambda_{1} \frac{\partial u_{1}^{M_{1}}(x)}{\partial n_{1}}=\Lambda_{1} u_{2}^{N_{1}}+\lambda_{1} \frac{\partial u_{2}^{N_{1}}(x)}{\partial n_{1}}, \forall x \in \Gamma_{1}
\end{aligned}
$$

3: Solve

$$
\begin{aligned}
L(x) u_{2}^{M_{2}}(x) & =f(x), \forall x \in \Omega_{2}, \\
u_{2}^{M_{2}}(x) & =g(x), \forall x \in \partial \Omega_{2} \backslash \Gamma_{2}, \\
\Lambda_{2} u_{2}^{M_{2}} & +\lambda_{2} \frac{\partial u_{2}^{M_{2}}(x)}{\partial n_{2}}=\Lambda_{2} u_{1}^{N_{2}}+\lambda_{2} \frac{\partial u_{1}^{N_{2}}(x)}{\partial n_{2}}, \forall x \in \Gamma_{2} .
\end{aligned}
$$

4: Enddo

According to the specific choice of the operators $\Lambda_{i}$ and the values of scalars $\lambda_{i}$, we obtain in Table 1 the family of Schwarz DD techniques. If $\Lambda_{1}=\Lambda_{2}=I d$ and $\lambda_{1}=\lambda_{2}=0$ then the above multiplicative version is the classical Multiplicative Schwarz. If $\Lambda_{1}=\Lambda_{2}=$ constant $>0$ and $\lambda_{1}=\lambda_{2}=1$ then it is the modified Schwarz proposed by Lions in [25]. 


\begin{tabular}{|c|c|c|c|c|c|}
\hline Overlap & $\Lambda_{1}$ & $\Lambda_{2}$ & $\lambda_{1}$ & $\lambda_{2}$ & Method \\
\hline yes & $I d$ & $I d$ & 0 & 0 & Schwarz \\
\hline yes & $I d$ & $I d$ & $\alpha$ & $\alpha$ & ORAS [36] \\
\hline No & $I d$ & 0 & 0 & 1 & Neumann-Dirichlet [26] \\
\hline No & $I d$ & $I d$ & 1 & 1 & Modified Schwarz [24] \\
\hline
\end{tabular}

TABLE 1. Derived methods obtained from the specific choices of the operators $\Lambda_{i}$ and the values of scalars $\lambda_{i}$ in the GSAM.

\subsection{Convergence property of the GSAM}

Engquist \& Zhao [12] showed that with an appropriate choice of the operators $\Lambda_{i}$ this DD method converges in two iterations. They established the proposition that follows:

Proposition 1.1. If $\Lambda_{1}$ (or $\Lambda_{2}$ ) is the Dirichlet to Neumann operator at the artificial boundary $\Gamma_{1}$ (or $\Gamma_{2}$ ) for the corresponding homogeneous PDE in $\Omega_{2}$ (or $\Omega_{1}$ ) with homogeneous $B C$ on $\partial \Omega_{2} \cap \partial \Omega$ (or $\partial \Omega_{1} \cap \partial \Omega$ ) then the Generalized Schwarz Alternating method converges in two steps.

The GSAM method converges in two steps if the Dirichlet-Neumann operators $\Lambda_{i}, i=1,2$, are available. These operators are not local to a sub-domain but they link up together all the sub-domains if their number is greater than two.

In the Aitken-Schwarz methodology introduced by $[17,18]$, only the purely linear convergence property of the Schwarz method (for local linear operator in subdomain) is used. Consequently, no direct approximation of the Dirichlet-Neumann map is used, but an approximation of the operator of error linked to this Dirichlet-Neumann map is performed.

The pure linear convergence of the GSAM can be established as follows. Define the projection operator $P_{i}: H^{1}\left(\Omega_{i}\right) \rightarrow H^{1}\left(\Omega_{i i}\right)$ to be the restriction from $\Omega_{i}$ to $\Omega_{i i}$. Denote $S_{i}$ (respectively $S_{i i}$ ) to be the Dirichlet to Neumann map operator in $\Omega_{i}$ (respectively $\Omega_{i i}$ ) on $\Gamma_{i}$ (respectively $\Gamma_{\bar{i}}$ ). Let $R_{i}$ be the restriction operator from $H^{1}\left(\Omega_{i}\right)$ to $H^{1 / 2}\left(\Gamma_{i}\right)$ and $R_{i}^{*}$ be the right inverse operator of $R_{i}$, i.e, $R_{i} R_{i}^{*}=I, \forall g \in H^{1 / 2}\left(\Gamma_{i}\right), L(x) R_{i}^{*} g=$ $0, R_{i}^{*} g=g$ on $\Gamma_{i}, R_{i}^{*} g=0$ on $\partial \Omega_{i} \backslash \Gamma_{i}$. Let $R_{i i}$ be the corresponding operator in $\Omega_{i i}, \forall g \in H^{1 / 2}\left(\Gamma_{\bar{i}}\right), L(x) R_{i i}^{*} g=$ $0, R_{i i}^{*} g=g$ on $\Gamma_{\bar{i}}, R_{i i}^{*} g=0$ on $\partial \Omega_{i i} \backslash \Gamma_{\bar{i}}$

The following proposition (see [[12],p.354]) establishes the convergence of the GSAM.

Proposition 1.2. After one cycle of iteration on the error function $e_{i}^{n}=u-u_{i}^{n}$ in $\Omega_{i}$ for the GSAM, we have for $\{i, j\}=\{1,2\}, i \neq j$ :

$$
e_{i}^{n}=R_{i}^{*}\left(\Lambda_{i}+\lambda_{i} S_{i}\right)^{-1}\left(\Lambda_{i}-\lambda_{i} S_{j j}\right) R_{j j} P_{j} R_{j}^{*}\left(\Lambda_{j}+\lambda_{j} S_{j}\right)^{-1}\left(\Lambda_{j}-\lambda_{i} S_{i i}\right) R_{i i} P_{i}\left(e_{i}^{n-2}\right) .
$$

For the non-overlapping Schwarz $\left(\Gamma_{1}=\Gamma_{2}, P_{i}=I d, R_{i i}=R_{i}\right)$, Eq (21) is reduced to

$$
e_{i}^{n}=R_{i}^{*}\left(\Lambda_{i}+\lambda_{i} S_{i}\right)^{-1}\left(\Lambda_{i}-\lambda_{i} S_{j}\right)\left(\Lambda_{j}+\lambda_{j} S_{j}\right)^{-1}\left(\Lambda_{j}-\lambda_{j} S_{i}\right) R_{i} e_{i}^{n-1} .
$$

Consequently, by applying $R_{i}$ to Eq $(22)$ we see that the trace of the error on $\Gamma_{i}$ satisfies:

$$
R_{i} e_{i}^{n}=\underbrace{R_{i} R_{i}^{*}}_{I d} \underbrace{\left(\Lambda_{i}+\lambda_{i} S_{i}\right)^{-1}\left(\Lambda_{i}-\lambda_{i} S_{j}\right)\left(\Lambda_{j}+\lambda_{j} S_{j}\right)^{-1}\left(\Lambda_{j}-\lambda_{j} S_{i}\right)}_{P} R_{i} e_{i}^{n-1} .
$$

The trace of the error converges purely linearly as the error operator $P$ does not depend of the Schwarz iteration. The Aitken-Schwarz methodology uses this property to accelerate the convergence of the Schwarz algorithm to the right solution at the artificial interface.

Remarque 1.3. The same convergence applies in the general case of GSAM with applying the operator $R_{i i} P_{i}$ on both sides of Eq (21). 


\subsection{Connexion between Steklov-Poincaré and Schur complement operators}

For simplicity, let us consider two subdomains with no overlap. Then the discretization of Eq (3), with either finite volume or second order finite differences, leads to the following matrix system:

$$
A u=\left(\begin{array}{ccc}
A_{11} & 0 & A_{13} \\
0 & A_{22} & A_{23} \\
A_{31} & A_{32} & A_{33}^{(1)}+A_{33}^{(2)}
\end{array}\right)\left(\begin{array}{l}
u_{1} \\
u_{2} \\
u_{3}
\end{array}\right)=\left(\begin{array}{c}
f_{1} \\
f_{2} \\
f_{3}^{(1)}+f_{3}^{(2)}
\end{array}\right)
$$

where $u_{1}$ and $u_{2}$ denote the vector of interior unknowns in sudomains $\Omega_{1}$ and $\Omega_{2}$, respectively, and $u_{3}$ denotes the vector of remaining nodal unknowns on $\Gamma$. The interior nodal unknowns can be formally eliminated from Eq (23) by writting:

$$
\begin{aligned}
& u_{1}=A_{11}^{-1}\left(f_{1}-A_{13} u_{3}\right), \\
& u_{2}=A_{22}^{-1}\left(f_{2}-A_{23} u_{3}\right),
\end{aligned}
$$

and by denoting

$$
\begin{aligned}
& S_{1}=A_{33}^{(1)}-A_{31} A_{11}^{-1} A_{13}, g_{1}=f_{3}^{(1)}-A_{31} A_{11}^{-1} f_{1}, \\
& S_{2}=A_{33}^{(2)}-A_{32} A_{22}^{-1} A_{23}, g_{2}=f_{3}^{(2)}-A_{32} A_{22}^{-1} f_{2},
\end{aligned}
$$

we obtain the interface Schur complement system

$$
S u_{3}=\left(S_{1}+S_{2}\right) u_{3}=g_{1}+g_{2}=g
$$

Note that the following identity holds (see [27]):

$$
\hat{A}_{11}\left(\begin{array}{c}
0 \\
S_{1} x_{3}
\end{array}\right)=\left(\begin{array}{cc}
A_{11} & A_{13} \\
A_{31} & A_{33}^{(1)}
\end{array}\right)^{-1}\left(\begin{array}{c}
0 \\
S_{1} u_{3}
\end{array}\right)=\left(\begin{array}{cc}
A_{11} & A_{13} \\
0 & I
\end{array}\right)^{-1}\left(\begin{array}{c}
0 \\
u_{3}
\end{array}\right)
$$

The matrix $\hat{A}_{11}$ corresponds to the discrete stiffness matrix on subdomain $\Omega_{1}$ with homogeneous Dirichlet BC on the external boundary segment and Neumann BC on the interface boundary $\Gamma$. So that, the matrix vector product $S_{1} x_{3}$ is simply the discrete conormal derivative on $\Gamma$ associated with the homogeneous equivalent of Eq(14) on the sub-domain $\Omega_{1}$ with zeros boundary data on $\partial \Omega_{1} \backslash \Gamma$ and prescribed non zeros values $u_{3}$ on $\Gamma$ (i.e for $\left.S_{2} u_{3}\right)$. These matrix vector products are the discrete equivalents of the Steklov-Poincaré operator on the respective subdomains.

At the discrete level the pure linear convergence of the GSAM holds, and the operator error $P$ on trace error involves the local Schur complement of al the subdomains. In practice, some algebraic approximations of these local Schur complements are used (see $[8,15,19]$ ), while in the Aitken-Schwarz methodology, we do not search to approximate the local Schur complement but to build the discrete matrix $P$ or an approximation of this matrix $P$, in order to accelerate the convergence.

\section{Aitken-Schwarz Method for Linear Operators}

The pure linear convergence property of the generalized Schwarz alternating method suggests that the convergence sequence of traces solution at the artificial interfaces can be accelerated by the well known process of Aitken acceleration of convergence. In fact, the radius of the interface error operator doesn't impact the process and even a divergent algorithm can be accelerated if it diverges purely linearly. Thus the optimal choice of the GSAM parameters is not needed. 


\subsection{Aitken-Schwarz 1D problem case}

In the case of the additive GSAM $\left(\Lambda_{1}=\Lambda_{2}=I d, \lambda_{1}=\lambda_{2}=0\right)$ with two overlapping subdomains, the iterations of the errors at the interfaces can be written as follows:

$$
\left(\begin{array}{c}
e_{1}^{n+1}\left(\Gamma_{2}\right) \\
e_{2}^{n+1}\left(\Gamma_{1}\right)
\end{array}\right)=\left(\begin{array}{cc}
0 & \delta_{2} \\
\delta_{1} & 0
\end{array}\right)\left(\begin{array}{c}
e_{1}^{n}\left(\Gamma_{2}\right) \\
e_{2}^{n}\left(\Gamma_{1}\right)
\end{array}\right)
$$

Consequently, if $\delta_{1}, \delta_{2}$ are not known, they can be deduced from the relation:

$$
\left(\begin{array}{cc}
e_{1}^{n+2}\left(\Gamma_{2}\right) & e_{1}^{n+1}\left(\Gamma_{2}\right) \\
e_{2}^{n+2}\left(\Gamma_{1}\right) & e_{2}^{n+1}\left(\Gamma_{1}\right)
\end{array}\right)\left(\begin{array}{ll}
e_{1}^{n+1}\left(\Gamma_{2}\right) & e_{1}^{n}\left(\Gamma_{2}\right) \\
e_{2}^{n+1}\left(\Gamma_{1}\right) & e_{2}^{n}\left(\Gamma_{1}\right)
\end{array}\right)^{-1}=\left(\begin{array}{cc}
0 & \delta_{2} \\
\delta_{1} & 0
\end{array}\right)=P
$$

If $\delta_{1} \delta_{2} \neq 1$, the Aitken acceleration gives the exact solution at the interfaces :

$$
\begin{aligned}
& u^{\infty}\left(\Gamma_{1}\right)=\left[-u_{2}^{1}+\delta_{2} u_{1}^{0}+\delta_{2}\left(\delta_{1} u_{2}^{0}-u_{1}^{1}\right)\right] /\left(\delta_{1} \delta_{2}-1\right), \\
& u^{\infty}\left(\Gamma_{2}\right)=\left[-u_{1}^{1}+\delta_{1} u_{2}^{0}+\delta_{1}\left(\delta_{2} u_{1}^{0}-u_{2}^{1}\right)\right] /\left(\delta_{1} \delta_{2}-1\right)
\end{aligned}
$$

An additional solve of each of the subproblems with BCs $u_{\Gamma_{i}}^{\infty}$ gives the solution. The Aitken acceleration thus transforms the additive Schwarz procedure into an exact solver regardless of the speed of convergence of the original Schwarz method. We observe that $\delta_{1}, \delta_{2}$ are dependent only on the operator and the partitioning of the domain. $\delta_{i}, i=\{1,2\}$ for example can be computed before hand as follows. Let $v_{i}$ be the solution of

$$
L(x) v_{i}(x)=0, \forall x \in \Omega_{i}, \text { with } v_{i \mid \Gamma_{i}}=1 .
$$

We have then $\delta_{\bar{i}}=v_{i \mid \Gamma_{\bar{i}}}$. $v_{i}$ can be computed numerically and possibly analytically if the differential operator $L(x)$ is simple enough. When $\delta_{i}, i=\{1,2\}$ is known, we need only one Schwarz iterate to accelerate the interface and an additional solve for each subproblem. This is a total of two solves per sub-domain.

\subsection{The Aitken-Schwarz method on linear separable operators and regular mesh on ar- tificial interface}

In order to show the case with several subdomains, consider the 3D Poisson equation, where the domain is discretized with regular size steps in each direction, and the separable Poisson operator is approximated using second order finite differences. We used additive Schwarz with Dirichlet BC $\left(\Lambda_{i}=I d\right.$ and $\lambda_{i}=0$ in GSAM). The overlap is set to the minimum of one cell or one step size as the Aitken-Schwarz method is not related to the rate of convergence but only to the pure linear convergence or divergence property. As we use a regular mesh at the artificial interfaces, we can proceed as previously with writing the solution on the artificial interfaces generated by the Schwarz algorithm in a sine expansion in the $y$ and $z$ directions. Thus for:

$$
\left\{\begin{array}{l}
-\left(\frac{\partial^{2}}{\partial x^{2}}+\frac{\partial^{2}}{\partial y^{2}}+\frac{\partial^{2}}{\partial z^{2}}\right) u(x, y, z)=f(x, y, z), \forall(x, y, z) \in \Omega=[0, \pi]^{3} \\
u(x, y, z)=0, \forall(x, y, z) \in \partial \Omega
\end{array}\right.
$$

we search for $u(x, y, z)=\sum_{1 \leq j \leq n_{y}} \sum_{1 \leq k \leq n_{z}} \hat{u}_{j k}(x) \sin (j y) \sin (k z)$. The $3 \mathrm{D}$ problem is equivalent to the following $n_{y} \times n_{z}$ discrete problems:

$$
\left\{\begin{array}{l}
\frac{-\hat{u}_{j k, i+1}+2 \hat{u}_{j k, i}-\hat{u}_{j k, i-1}}{h_{x}^{2}}+\lambda_{j} \hat{u}_{j k, i}+\lambda_{k} \hat{u}_{j k, i}=\hat{f}_{j k, i}, 1 \leq i \leq N_{x}-1 \\
\hat{u}_{j k, 0}=\alpha_{j k} \\
\hat{u}_{j k, n_{x}}=\beta_{j k}
\end{array}\right.
$$


where $\lambda_{j}=\frac{4}{h_{y}^{2}} \sin ^{2}\left(\frac{j h_{y}}{2}\right)$ (respectively $\lambda_{k}=\frac{4}{h_{z}^{2}} \sin ^{2}\left(\frac{k h_{z}}{2}\right)$ ) are the eigenvalues of the $\frac{\partial^{2}}{\partial y^{2}}$ (respectively $\frac{\partial^{2}}{\partial z^{2}}$ ) operator with homogeneous BC approximated by a second order finite differences scheme with a regular step size $h_{y}$ (respectively $h_{z}$ ). $h_{x}$ represents the regular step size in the $x$ direction.

The domain in the $x$ direction is split in $q$ subdomains $\Omega_{i}=\left(X_{i}^{l}, X_{i}^{r}\right), i=1 . . q$ with $X_{2}^{l}<X_{1}^{r}<X_{3}^{l}<$ $X_{2}^{r}, \ldots, X_{q}^{l}<X_{q-1}^{r}$. For the sake of simplicity we choose the subdomains $\Omega_{i}$ to have the same size, discretized with a regular step size $h_{x}=\frac{X_{i}^{l}-X_{i}^{r}}{n_{x}}$ given $n_{x}+1$ points $x_{l}=X_{i}^{l}+l h_{x}$. Then the discrete error $\hat{e}_{i}$ of the Schwarz algorithm on subdomain $\Omega_{i}$ for the mode $j k$ at iterate $n+1$ satisfies :

$$
\left\{\begin{array}{l}
\hat{e}_{i}^{n+1}\left(x_{l+1}\right)-\left(2+\lambda_{j} h_{x}+\lambda_{k} h_{x}\right) \hat{e}_{i}^{n+1}\left(x_{l}\right)+\hat{e}_{i, l-1}^{n+1}\left(x_{l-1}\right)=0,1 \leq l \leq n_{x}-1, \\
\hat{e}_{i}^{n+1}\left(X_{i}^{l}\right)=\hat{e}_{i-1}^{n}\left(X_{i}^{l}\right) \\
\hat{e}_{i}^{n+1}\left(X_{i}^{r}\right)=\hat{e}_{i+1}^{n}\left(X_{i}^{r}\right) .
\end{array}\right.
$$

The generic solution of this system of linear equations writes:

$$
\hat{e}_{i}^{n+1}\left(x_{l}\right)=C_{1} Z_{1}^{l}+C_{2} Z_{2}^{l}, 0 \leq l \leq n_{x}
$$

where $Z_{1}$ and $Z_{2}$ are the roots of $-Z^{2}+\left(2+\lambda_{j} h_{x}^{2}+\lambda_{k} h_{x}^{2}\right) Z-1=0$. The two constant parameters $C_{1}$ and $C_{2}$ are given by the BCs:

$$
\left(\begin{array}{cc}
1 & 1 \\
Z_{1}^{n_{x}} & Z_{2}^{n_{x}}
\end{array}\right)\left(\begin{array}{c}
C_{1} \\
C_{2}
\end{array}\right)=\left(\begin{array}{c}
\hat{e}_{i-1}^{n}\left(X_{i}^{l}\right) \\
\hat{e}_{i+1}^{n}\left(X_{i}^{r}\right)
\end{array}\right)
$$

Thus the error on domains $\Omega_{i-1}$ and $\Omega_{i+1}$ at iterate $n+1$ depends on the error at iterate $n$ on domain $\Omega_{i}$. One can write:

$$
\left(\begin{array}{c}
\hat{e}_{i-1}^{n+1}\left(X_{i-1}^{r}\right) \\
\hat{e}_{i+1}^{n+1}\left(X_{i+1}^{l}\right)
\end{array}\right)=\left(\begin{array}{cc}
\delta_{i}^{l, l} & \delta_{i}^{l, r} \\
\delta_{i}^{r, l} & \delta_{i}^{r, r}
\end{array}\right)\left(\begin{array}{c}
\hat{e}_{i}^{n}\left(X_{i}^{l}\right) \\
\hat{e}_{i}^{n}\left(X_{i}^{r}\right)
\end{array}\right)
$$

Then coefficient $\delta_{i}^{l, l}$ (respectively $\left.\delta_{i}^{r, l}\right)$ is the value of $\hat{e}_{i}^{n}\left(X_{i-1}^{r}\right)$ (respectively $\left(\hat{e}_{i}^{n}\left(X_{i+1}^{l}\right)\right)$ when the BCs on domain $\Omega_{i}$ are $\hat{e}_{i}^{n}\left(X_{i}^{l}\right)=1$ and $\hat{e}_{i}^{n}\left(X_{i}^{r}\right)=0$ while coefficient $\delta_{i}^{l, r}$ (respectively $\delta_{i}^{r, r}$ ) is the value $\hat{e}_{i}^{n}\left(X_{i-1}^{r}\right)$ (respectively $\left.\hat{e}_{i}^{n}\left(X_{i+1}^{l}\right)\right)$ when the BCs on domain $\Omega_{i}$ are $\hat{e}_{i}^{n}\left(X_{i}^{l}\right)=0$ and $\hat{e}_{i}^{n+1}\left(X_{i}^{r}\right)=1$. Consequently, these values can be found analytically once for all by computing the values $C_{1}$ and $C_{2}$ associated to the BCs and by evaluating the function $\hat{e}_{i}^{n}(x)$ in $X_{i-1}^{r}$ and $X_{i+1}^{l}$. If we choose a minimum overlap of $h_{x}$ between subdomains $\left(X_{i-1}^{r}=X_{i}^{l}+h_{x}\right.$ and $\left.X_{i+1}^{l}=X_{i}^{r}-h_{x}\right)$ we obtain:

$$
P_{i} \stackrel{\text { def }}{=}\left(\begin{array}{cc}
\delta_{i}^{l, l} & \delta_{i}^{l, r} \\
\delta_{i}^{r, l} & \delta_{i}^{r, r}
\end{array}\right)=\left(\begin{array}{cc}
\frac{Z_{1} Z_{2}\left(Z_{2}^{n_{x}-1}-Z_{1}^{n_{x}-1}\right)}{Z_{2}^{n_{x}}-Z_{1}^{n_{x}}} & \frac{\left(Z_{1} Z_{2}\right)^{n_{x}-1}\left(Z_{2}-Z_{1}\right)}{Z_{2}^{n_{x}}-Z_{1}^{n_{x}}} \\
\frac{Z_{2}-Z_{1}}{Z_{2}^{n_{x}}-Z_{1}^{n_{x}}} & \frac{Z_{2}^{n_{x}-1}-Z_{1}^{n_{x}-1}}{Z_{2}^{n_{x}}-Z_{1}^{n_{x}}}
\end{array}\right)
$$

The matrix $P_{i}$ can also be computed numerically from three iterations as

$$
P_{i}=\left(\begin{array}{cc}
\hat{e}_{i-1}^{n+2}\left(X_{i-1}^{r}\right) & \hat{e}_{i-1}^{n+1}\left(X_{i-1}^{r}\right) \\
\hat{e}_{i+1}^{n+2}\left(X_{i+1}^{l}\right) & \hat{e}_{i+1}^{n+1}\left(X_{i+1}^{l}\right)
\end{array}\right)\left(\begin{array}{cc}
\hat{e}_{i}^{n+1}\left(X_{i}^{l}\right) & \hat{e}_{i}^{n}\left(X_{i}^{l}\right) \\
\hat{e}_{i}^{n+1}\left(X_{i}^{r}\right) & \hat{e}_{i}^{n}\left(X_{i}^{r}\right)
\end{array}\right)^{-1}
$$

where $e_{i}^{n+2}=u_{i}^{n+2}-u_{i}^{n+1}$

We note $e_{i}^{l, n}=e_{i}^{n}\left(X_{i}^{l}\right), e_{i}^{r, n}=e_{i}^{n}\left(X_{i}^{r}\right)$ and $\tilde{e}^{n}$ the $n^{\text {th }}$ iterated error restricted at the interfaces, i.e

$$
\tilde{e}^{n}=\left(e_{2}^{l, n}, e_{1}^{r, n}, e_{3}^{l, n}, e_{2}^{r, n}, \ldots, e_{q}^{l, n}, e_{q-1}^{r, n}\right)^{T}
$$


Let $P$ be the matrix that links the error on artificial interfaces of the Schwarz DD. $P$ has the following pentadiagonal structure:

From the equality

$$
\begin{array}{|cccccccc}
0 & \delta_{1}^{r} & 0 & 0 & \ldots & & & \\
\delta_{2}^{l, l} & 0 & 0 & \delta_{2}^{l, r} & \ldots & & & \\
\delta_{2}^{r, l} & 0 & 0 & \delta_{2}^{r, r} & \ldots & & & \\
& & & & & & & \\
& & & \ldots & \delta_{q-1}^{l, l} & 0 & 0 & \delta_{q-1}^{l, r} \\
& & & \ldots & \delta_{q-1}^{r, l} & 0 & 0 & \delta_{q-1}^{r, r} \\
& & & \ldots & 0 & 0 & \delta_{q}^{r} & 0
\end{array} \mid
$$

$$
\tilde{e}^{n+1}=P\left(\tilde{e}^{n}\right)
$$

one writes the Aitken acceleration as follows:

$$
\tilde{u}^{\infty}=(I d-P)^{-1}\left(\tilde{u}^{n+1}-P \tilde{u}^{n}\right)
$$

If the additive Schwarz method converges, then $\|P\|<1$ and $I d-P$ is non singular.

Let us report the results obtained to solve the Poisson equation on a metacomputing framework based on networks that provide as low a level of performance as Ethernet [2]. For large scale metacomputing experiments, we are using up to three Cray T3E located in Stuttgart, Pittsburgh, and Jülich with 512 processors cadenced to $450 \mathrm{Mhz}, 40 \mathrm{Mhz}$ and $375 \mathrm{Mhz}$, a latency of $12 \mu \mathrm{s}$ and a communication bandwidth of $320 \mathrm{MB} / \mathrm{s}$.

The main challenge is to combine the Aitken-Schwarz DD method with an efficient parallel subdomain solver as PDC3D in order to have a highly efficient solver for the 3D Poisson operator for metacomputing environments. PDC3D [34],[33], is a parallel fast direct solution method for linear systems with separable block tridiagonal matrices. The method under consideration has the arithmetical complexity $\mathcal{O}\left(N \log ^{2} N\right)$, and is closely related to the cyclic reduction method. This very efficient solver requires a high performance communication network mainly due to the necessary global reduction operations, to gather the partial solution. It reaches a very good scalability for a CrayT3E.

For all our experiments, the bandwidth fluctuated in the range of $1.6-5 \mathrm{Mb} / \mathrm{s}$ and the latency was about $30 \mathrm{~ms}$. We used the PACX-MPI communication library [7] that used the best communication network available for exchange of data between processors. The mpi vendor implementation is used when two nodes of a same machine must communicate and MPI with TCP-IP protocol is used in a user transparent way when two nodes of different machines have to communicate.

We have a three-dimensional DD and a two-level algorithm combining Aitken-Schwarz in the $x$-direction and PDC3D solver in the $y$ and $z$ directions for subdomain problems local to machines (named macro) that matches the hierarchy of the network speed and access to memory speed in a distributed GRID environment. The number of grid points in Aitken DD direction $x$ is balanced in such a way that PDC3D requires the same CPU time on each system. We defined the barrier between low and medium size frequencies in each space variable to be $1 / 4$ of the number of waves. We did not accelerate the upper half of the frequencies. We checked that the impact on the numerical error against an exact polynomial solution is in the interval $\left[10^{-7}, 10^{-6}\right]$ for our test cases with minimum overlap between macro subdomains. In [2] it is shown the good scalability performance of the method on one single Cray T3E with requiring no more than 6 seconds to solve the problem with $46 \times 10^{6}$ unknowns with 256 processors running at $450 \mathrm{MHz}$. Same results of scalability have been obtained in the metacomputing experiment using the machines CrayN, CrayS (see Figure 1). Adding the CrayP and running on 1280 processors in total we solved a problem with 700 million unknowns in 62 seconds. As we have a methodology that scales well, even in a metacomputing framework, for linear separable operator on regular mesh, our objective is now to extend the methodology for linear operator strongly non separable and non regular mesh. The difficulty is the same as the non regular mesh or the non separability will link the components of the error together and made the matrix $P$ full. 
Extensibility in metacomputing versus macrosubdomains

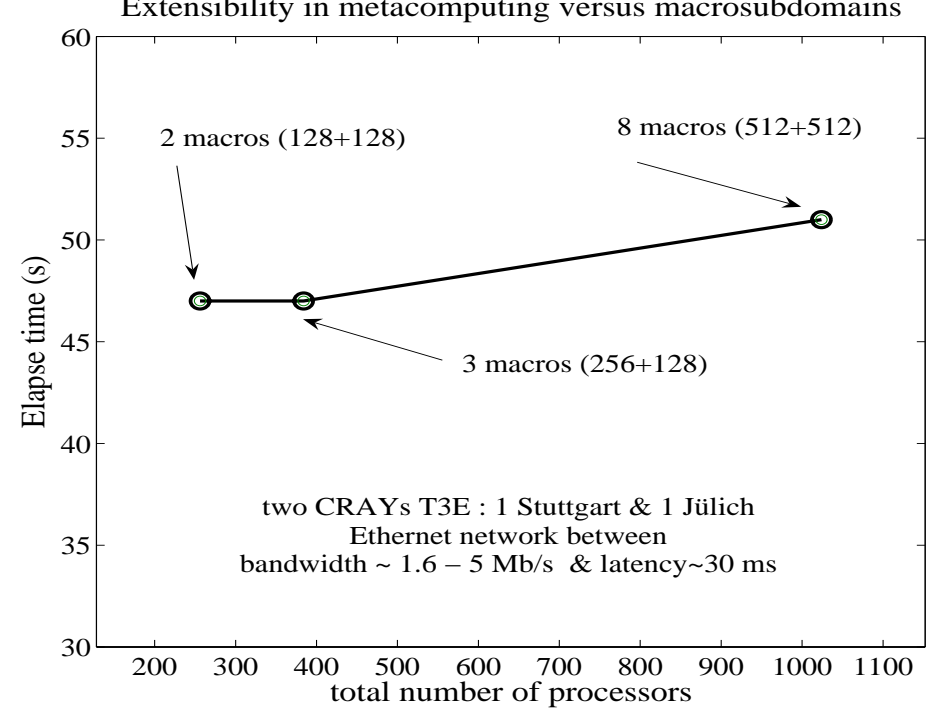

FiguRe 1. Scalability of the Aitken Schwarz algorithm for the 3D Poisson problem in various metacomputing environments with CrayN and CrayS.

\subsection{The Aitken-Schwarz method for linear non separable operators and/or non regular mesh on artificial interface}

For non-regular interface discretization the classical Fourier transform is not available. Some generalizations of the Fourier approach have been proposed in [16] and [1].

In [1] for a separable operator on a non regular mesh, an eigenvalue decomposition of the part of the operator acting in one direction is performed. Then the eigenvectors of this eigen-decomposition are used as generalized Fourier modes, the matrix $P$ associated to each eigenvector being computed as in the regular mesh case by decomposing the solution trace on the interface in this eigenvector basis and then accelerating each generalized Fourier mode by Aitken's formula before coming back in the "physical" space to have the accelerated solution on the interface.

In [16], the eigen-pair is computed on the trace transfer operator $P$ which links the Schwarz iterate errors on the interface. Then three algorithms are proposed:

a a bandwidth approximation of matrix $P$ : a block diagonal approximation of matrix $P$ is performed and then the eigen-pair of this matrix block is searched,

b a coarse grid approximation of matrix $P$ : it consists in defining a coarse grid and having restriction $T_{h / H}$ and projection $T_{H / h}$ to exchange between the fine grid and the coarse grid and then defining the coarse approximation of matrix $P$ as $T_{h / H} P T_{H / h}$,

c a compact representation of the interface: a least square approximation of the Schwarz iterates solution is done on a regular mesh where a sine or cosine expansion is performed, depending on the BC.

Some accelerations have been obtained on finite volume with approach (b), better results come from (c) with very few modes in the expansion even if the convergence seems to retrieve the original rate of convergence after the first acceleration. Strategy (a) works quite well with a band approximation of matrix $P$ of size $2 Z+1$ with $Z=\{1,2,3\}$ on a regular grid with a convection-diffusion operator closed to the Laplacian for which the matrix $P$ is diagonal. Nevertheless, the eigenvalue problem is very sensitive to perturbations of the matrix entries. Consequently, not taking into account a part of the matrix $P$ can greatly deteriorate the computed eigen base 
to represent the property of pure linear of the sequence of iterate solution.

\section{Non Uniform Discrete Fourier Transform}

We developed an original Non Uniform Discrete Fourier Transform (NUDFT) based on the values of the iterated solution at the interface mesh points. The most important feature of this approximation is that it provides a framework to perform adaptively the acceleration.

\subsection{Aitken-Schwarz in a multidimensional framework}

Let us consider the multidimensional case with the discretized version of the problem (3). We restrict ourselves for simplicity to the two overlapping subdomains case and the multiplicative GSAM algorithm. In the multidimensional framework the main difficulty consists in defining a representation of the interface solutions that can produce a cheap approximation of the error transfer operator matrix $P$.

For a linear operator one can write the sequence of Schwarz iterates $U_{\Gamma_{h}^{1}}^{0}, U_{\Gamma_{h}^{2}}^{0}, U_{\Gamma_{h}^{1}}^{1}, U_{\Gamma_{h}^{2}}^{1}, \ldots$, then the error between two Schwarz iterates behaves linearly. Errors $e_{\Gamma_{h}^{j}}^{i}=U_{\Gamma_{h}^{j}}^{i+1}-U_{\Gamma_{h}^{j}}^{i}$ satisfy

$$
\left(\begin{array}{c}
e_{\Gamma_{h}^{1}}^{i+1} \\
e_{\Gamma_{h}^{2}}^{i+1}
\end{array}\right)=P\left(\begin{array}{c}
e_{\Gamma_{h}^{1}}^{i} \\
e_{\Gamma_{h}^{2}}^{i},
\end{array}\right)
$$

where $P$ represents the iteration error operator at artificial interfaces.

Let us consider now a discretisation of the interfaces with the meshes $\Gamma_{h}^{j}$ and let us denote $\Gamma_{h}$ to be the coarsest discretisation in the sense that it produces the smallest set of orthogonal vectors $\Phi_{k}$ that belong to $\Gamma_{h}$ with respect to a discrete Hermitian form [[.,.]]. One can consider without loss of generality that these vectors satisfy $\left[\left[\Phi_{k}, \Phi_{k}\right]\right]=1, \forall k \in\{0, \ldots, N\}$. Being given $f \in C^{\infty}(\Gamma)$ then one can define the vector $F\left(\Gamma_{h}\right)$ of discrete values of a function $f$ on $\Gamma_{h}$. The set of vectors $\Phi_{k}$ defined on $\Gamma_{h}$ should also satisfy a density property in the space of $C^{\infty}(\Gamma)$ functions when $N \rightarrow \infty$. I.e. $\forall \epsilon>0, \exists N \in \mathbb{N}$ such that for $n \geq N$ the vector $F_{n}=\sum_{k=0}^{n} \alpha_{k} \Phi_{k}$ satisfies $\left\|F\left(\Gamma_{h}\right)-F_{n}\right\|_{\infty}<\epsilon$. Without this density property the acceleration of the convergence will converge only to the part of the solution interface that can be approximated by the set of $\Phi_{k}$. If the size of the set of orthogonal vectors $\Phi_{k}$ is the size of the discrete interface then we have an exact (algebraic) approximation.

Consider the decomposition of the trace of the Schwarz solution in this orthogonal basis $U_{\Gamma}$. Let $U_{\Gamma_{h}}$ be the decomposition of $U_{\Gamma}$ with respect to the orthogonal basis $\Phi$.

$$
U_{\Gamma_{h}}=\sum_{k=0}^{N} \alpha_{k} \Phi_{k} .
$$

The $\alpha_{k}$ represent the Fourier-like coefficients of the solution with respect to the basis $\Phi$. The orthogonality of vectors $\Phi_{k}$ gives directly the value of $\alpha_{k}$ :

$$
\alpha_{k}=\left[\left[U_{\Gamma}, \Phi_{k}\right]\right] .
$$

Let us consider now the decomposition of the traces in the basis $\Phi$. Then the nature of the convergence does not change if we consider the error coefficients in the basis $\Phi$ instead of the error in the physical space. One can write an equivalent equation to $\mathrm{Eq}(43)$ but in the coefficient space. Let $\beta_{\Gamma_{h}^{j}}^{i}$ be the coefficients of the error $e_{\Gamma_{h}^{j}}^{i}$. Then, we can write in the coefficient space: 


$$
\left(\begin{array}{c}
\beta_{\Gamma_{h}^{1}}^{i+1} \\
\beta_{\Gamma_{h}^{2}}^{i+1}
\end{array}\right)=P_{[[., .]]}\left(\begin{array}{c}
\beta_{\Gamma_{h}^{1}}^{i} \\
\beta_{\Gamma_{h}^{2}}^{i}
\end{array}\right) .
$$

This matrix $P_{[[., .]]}$has the same size as the matrix $P$. Nevertheless, we have more flexibility to define some consistent approximation of this matrix, since we have access to a posteriori estimate based on the module value of the Fourier coefficients.

\subsection{Explicit building of $P_{[[., .]]}$}

The explicit building of $P_{[[., .,]}$consists in computing how the basis functions $\Phi_{k}$ are modified by one Schwarz iteration. Figure 2 describes the steps for constructing the matrix $P_{[[., .]]}$. Step (a) starts from the the basis function $\Phi_{k}$ and gets its value on the interface in the physical space. Then step (b) performs two Schwarz iterates with zero local right hand sides and homogeneous BCs on $\partial \Omega=\partial\left(\Omega_{1} \cap \Omega_{2}\right)$. Step (c) decomposes the trace solution on the interface in the basis $\Phi$. Thus we obtain the column $k$ of the matrix $P_{[[., .]]}$.

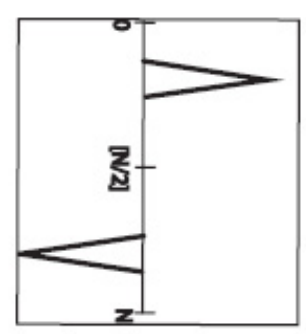

(a)

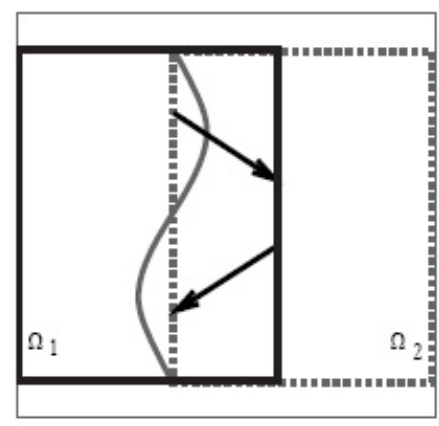

(b)

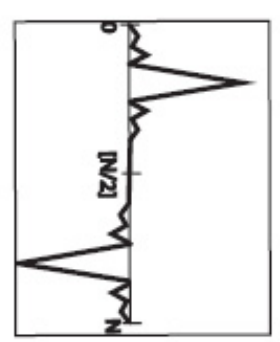

(c)

Figure 2. Steps to build the $P_{[[., .]]}$matrix

The full computation of $P_{[[., .]]}$can be done in parallel, but it needs as much local subdomain solves as the number of interface points (i.e the size of the matrix $P_{[[., .]]}$). Its adaptive computation is required to save computing time. The Fourier mode convergence gives a tool to select the Fourier modes that slow the convergence and have to be accelerated, contrary to the approach of [16], which builds an approximation of the operator in the physical space relatively to the trace solution on a coarse grid and then compute the Schur eigenvalue decomposition of this approximation. The adaptive Aitken-Schwarz algorithm can be described as follows:

\footnotetext{
Algorithm 2 Adaptative Aitken-Schwarz

1: Perform two GSAM iterates.

1: Write the difference between two successive iterates in the $\Phi$ basis and select the component modes higher than a fixed tolerance and with the worst convergence slope. Set Index the array of selected modes.

1: Take the subset $\tilde{v}$ of $m$ Fourier modes from 1 to $\max ($ Index).

2: Compute the $m \times m P^{*}{ }_{[[., .]]}$matrix associated to the $m$ modes selected which is an approximation of $P_{[[., .]]}$. 3: Accelerate the $m$ modes with the Aitken formula:

$$
\tilde{v}^{\infty}=\left(I d-P_{[[, .,]]}^{*}\right)^{-1}\left(\tilde{v}^{n+1}-P_{[[., .]]}^{*} \tilde{v}^{n}\right) .
$$

4: Recompose the solution with the $m$ mode accelerated and the $N-m$ other modes of the last iterate solution.
} 
The arithmetical computing cost is greatly reduced if $m \ll N$. It is possible to optimize the implementation by computing a number of columns of matrix $\tilde{P}_{[[., .]]}$during the Schwarz iteration on the problem. This considers a new local problem with several right hand sides (one of them corresponds to the initial problem, the others are associated to local problems to compute the columns of $\left.\tilde{P}_{[[., .]]}\right)$. The constraint is to obtain a resulting local problem that fits in the memory cache capacity. This can occur if there is a fast decay property of the components with the increase of the mode value as in the Fourier representation case. This paper will not focus on this aspect of the implementation because it depends on the hardware and on the considered problem.

\subsection{Fourier basis orthogonal with respect to a numerical Hermitian form}

For nonregular meshes, the conjugate property of the standard Fourier basis (see [4]) is lost. First, we gave a new definition of the Fourier basis in order to keep the conjugate property with respect to the nonregular mesh points:

Définition 3.1. Let $\left(x_{i}\right)_{0 \leq i \leq N}$ and $\left(z_{i}\right)_{0 \leq i \leq N}$ with $z_{i}=\frac{2 \pi i}{N}$ be two sets of points defined on $[0,2 \pi[$ such that $x_{i}=z_{i}+\epsilon_{i}$ with $(\epsilon)_{0 \leq i \leq N}$ defining the irregularity of the mesh.

Set $\psi_{l}(x)=\exp (i l x)_{0 \leq l \leq N / 2}$, then $\psi_{l}$ verify $\psi_{N-l}(x)=D^{N} \overline{\psi_{l}(x)}$, with $D=\operatorname{diag}\left(\epsilon_{i}\right)_{0 \leq i \leq N}$.

Define the functions:

$$
\phi_{l}(x)=\left\{\begin{array}{l}
\psi_{l}(x), 0 \leq l \leq N / 2 \\
D^{-N} \psi_{N-l}(x), N / 2+1 \leq l \leq N
\end{array}\right.
$$

Thus, the mesh sizes can vary from $\min _{i}\left(\epsilon_{i}\right)$ and $h_{r e f}+\max \left(\epsilon_{i}+\epsilon_{i+1}\right)$ with $h_{r e f}=\pi /(N-1)$. Then we define a numerical bilinear form that provides the orthogonality of the new Fourier basis. In this approach, we adopted the same point of view as [[4], p.89], that considers pseudospectral method as Galerkin method via a quadrature formula. To construct this numerical bilinear form, we must have as much equations as the orthogonality conditions. This leads to define the bilinear form as a symmetric Hermite quadrature formula that approximate the Fourier transform integral.

Définition 3.2. We introduce the following sesquilinear form in the space $S_{N}=\operatorname{span}\left\{\phi_{l}(x), 0 \leq l \leq N\right\}:$

$$
[[f, g]]=\sum_{l=0}^{N} \gamma_{l} f\left(x_{l}\right) \overline{g\left(x_{l}\right)}+\sum_{l=0}^{N} \beta_{l}\left(f^{\prime}\left(x_{l}\right) \overline{g\left(x_{l}\right)}+f\left(x_{l}\right) \overline{g^{\prime}\left(x_{l}\right)}\right) .
$$

The coefficients $\left\{\gamma_{l}\right\}_{l=0, \ldots, N}$ and $\left\{\beta_{l}\right\}_{l=0, \ldots, N}$ are chosen to satisfy the orthogonality condition among the basis functions $\left[\left[\phi_{l}, \phi_{k}\right]\right]=\delta_{l k}$, where $\delta_{l k}$ is the Kronecker symbol.

According to definition 3.1, this is done by solving the system of linear equations:

$$
\sum_{l=0}^{N} \gamma_{l} \exp \left(i k x_{l}\right)+\sum_{l=0}^{N} \beta_{l} i k \exp \left(i k x_{l}\right)=\delta_{0 k}, \quad k=-N, \ldots, N, \sum_{l=0}^{N} \beta_{l}=0 .
$$

Remarque 3.3. The condition number of this $2 N$ linear system depends on the $\epsilon_{i}$ values. Next section will provide some numerical tests on the condition number.

Then the nonuniform Fourier coefficients can be derived from the previous properties.

Définition 3.4. Given $\left(x_{i}\right)_{0 \leq i \leq N}$ as in definition 3.1 and $f=\left(\left(f_{i}\right)\right)_{0 \leq i \leq N}, f^{\prime}=\left(\left(f_{i}^{\prime}\right)\right)_{0 \leq i \leq N}$, the discrete Fourier coefficients of $f$ are given by:

$$
\tilde{f}_{k}=\left[\left[f, \Phi_{k}\right]\right], \quad k=-N / 2, \ldots, N / 2 .
$$


Or, in an algebraic framework:

$$
\begin{aligned}
\tilde{f} & =M_{1} f+M_{2} f^{\prime}, \quad M_{1}, M_{2} \in \mathbb{M}_{N+1}(\mathbb{C}) \\
M_{1}(k, l) & =\gamma_{l} \overline{\phi_{k}\left(x_{l}\right)}+\beta_{l} \overline{\phi_{k}^{\prime}\left(z_{l}\right)}, \\
M_{2}(k, l) & =\beta_{l} \overline{\phi_{k}\left(x_{l}\right)} .
\end{aligned}
$$

Proposition 3.5. With the notations of definitions 3.1-3.4, then the trigonometrical approximation

$$
\Pi_{N}^{F}(f(x))=\sum_{l=0}^{N} \tilde{f}_{k} \phi_{k}(x)
$$

where $\tilde{f}_{k}=\left[\left[f, \Phi_{k}\right]\right]$, with $f=\left(f\left(x_{i}\right)\right)_{0 \leq i \leq N}, f^{\prime}=\left(f^{\prime}\left(x_{i}\right)\right)_{0 \leq i \leq N} \in \mathbb{C}^{N+1}$, is exact for all $f \in \mathbf{T}^{N / 2}([0,2 \pi[)$, the space of trigonometrical polynomials of degree less or equal to $N / 2$.

Proof. Since $f \in \mathbf{T}^{N / 2}([0,2 \pi[)$, we can write it as a linear combination of the basis functions:

$$
f(x)=\sum_{k=-N / 2}^{N / 2} \eta_{k} \exp (i k x)=\sum_{m=0}^{N} \eta_{m} \Phi_{m}(x) .
$$

Now, since $[[\cdot, \cdot]]$ is sesquilinear

$$
\tilde{f}_{k}=\left[\left[f, \Phi_{k}\right]\right]=\sum_{m=0}^{N} \eta_{m}\left[\left[\Phi_{m}, \Phi_{k}\right]\right]=\sum_{m=0}^{N} \eta_{m} \delta_{m k}=\eta_{k} .
$$

Thus, the trigonometrical approximation of $f$ becomes:

$$
\Pi_{N}^{F}(f(x))=\sum_{l=0}^{N} \tilde{f}_{k} \phi_{k}(x)=\sum_{k=0}^{N} \eta_{k} \Phi_{k}(x)=f(x),
$$

which proves the proposition.

Proposition 3.6. For all $f \in C^{\infty}\left(\left[0,2 \pi[)\right.\right.$ there is a unique $f_{\Phi} \in \Phi$ which minimizes the distance of $f$ to $\Phi$.

Proof. Since $f \in C^{\infty}\left(\left[0,2 \pi[)\right.\right.$, we decompose $f$ in $f=f_{\Phi^{c}}+f_{\Phi}$ where $\Phi^{c}$ is the complementary of $\Phi$ in $C^{\infty}\left(\left[0,2 \pi[)\right.\right.$. As $f_{\Phi} \in \Phi$, one can write $f_{\Phi}=\sum_{m=0}^{N} \eta_{m} \Phi_{m}$. The coefficients $\eta_{m}$ are unique. Suppose there are two sets $\eta$ and $\tilde{\eta}$ that represent $f_{\Phi}$, then $\sum_{m=0}^{N}\left(\eta_{m}-\tilde{\eta}_{m}\right) \Phi_{m}=0$ with $\eta_{m} \neq \tilde{\eta}_{m}, \forall m$ which is not possible due to the linear independence of functions $\Phi_{m}$. Consequently $f$ admits a unique decomposition of the form $f=f_{\Phi^{c}}+f_{\Phi}$. Since [[.,.]] is positive definite on $\Phi$, it defines a scalar product on $\Phi$ an so $f_{\Phi}$ is the projection of $f$ on $\Phi$.

In applications vector $f$, which represents the values of a function $f(x)$ at the points $\left(x_{i}\right)_{0 \leq i \leq N}$, is given. No information is given on the vector $f^{\prime}$ which is needed in definition 3.4. In order to define the vector $f^{\prime}$ without differentiating vector $f$ that can deteriorate the exact approximation property demonstrated in proposition 3.5, we implicitly imposed the definition of $f^{\prime}$ with respect to the approximation polynomial property. This leads to a formulation of the Fourier approximation that depends only on the values of the function $f$ at the irregular mesh points. We set: 


$$
\frac{d}{d x}\left(\Pi_{N}^{F}(f(x))\right)_{\mid x=x_{l}}=f^{\prime}\left(x_{l}\right), \quad l=0, \ldots, N-1 .
$$

In an algebraic form, if we denote by $M_{\phi}$ the matrix whose elements are:

$$
M_{\phi}(l, k)=\phi_{k}^{\prime}\left(x_{l}\right)
$$

then the vector $f^{\prime}$ is obtained by solving the algebraic system:

$$
\left(i d_{N+1}-M_{\phi} M_{2}\right) f^{\prime}=M_{\phi} M_{1} f
$$

where $i d_{N+1}$ is the identity matrix in $\mathcal{M}_{N+1}(\mathbb{C})$.

\subsection{Computational cost}

The computational cost for this nonuniform Fourier transform can be split in two parts. The first part is the computing of the weight coefficients for the Hermitian quadrature formula. This cost is due once for all. According to the numerical solver used for the linear system, direct or Krylov solver, this cost is in $O\left((2 N)^{3}\right)$ or $O\left((2 N)^{2}\right)$. The second part consists in the "implicit" computing of the derivatives. This cost must be paid for each nonuniform Fourier transform. It costs $O\left(N^{2}\right)$ to $O\left(N^{3}\right)$ flops according to the iterative or direct solver used. These costs are greater than for the classical FFT on an uniform mesh which are in $O(N \log (N))$ in 1D and $O\left(N^{2} \log (N)\right)$ in $2 \mathrm{D}$. The FFT is based on the conjugate property of the Fourier basis in order to reduce the computation complexity. Here, we have also some conjugate property between the nonuniform Fourier basis. Nevertheless, the reduction of the computational cost remains under investigation.

\subsection{Numerical tests on nonuniform Fourier approximation}

Figure 3 gives the approximation property of the NUDFT with respect to the continuity of the approximated function and the number of points. The approximated solution is $f(x)=\frac{1}{\|f\|_{\infty}} \exp \left(-40\left(\frac{2}{3} \pi-x\right)^{2}\right)\left(\left(\frac{2}{3} \pi-x\right)^{2}\right)^{\frac{2 n+1}{2}}$ with $n \in\left\{-\frac{1}{2}, 0,1,2,3\right\}$. The test consists in computing the nonuniform Fourier coefficients and then in projecting the approximate solution on a regular grid of 1000 points and comparing the error with respect to the exact solution in the maximum norm. The results show the spectral convergence of the approximation with respect to the continuity of the function $f$. Moreover parameter $\epsilon$ has few impacts on the approximation until $h_{r e f} / 2$, while for $\epsilon=h_{r e f}$ the condition number involved in the computation of the Hermitian product weight is too high as table 2 shows, giving results concerning the interpolation property error with respect to the number of discretisation points and to the parameter $(\epsilon)$, for an analytical function $f(x)=\exp \left(-40(x-(2 \pi / 3))^{2}\right)$.

\begin{tabular}{|c||c|c|c|c|c|}
\hline $\mathrm{N}$ & $\epsilon=h_{u} / 2$ & $\epsilon=h_{u}$ & $\epsilon=2 h_{u}$ & $\epsilon=4 h_{u}$ & \\
\hline $2^{6}$ & $6.61 \mathrm{E}-4$ & $8.27 \mathrm{E}-4$ & $4.4 \mathrm{E}-3$ & $2.27 \mathrm{E}-2$ & $\left\|f-\Pi_{N}^{F}(f)\right\|_{\infty}$ \\
& $1.36 \mathrm{E}+3$ & $3.98 \mathrm{E}+4$ & $3.2 \mathrm{E}+8$ & $3.8 \mathrm{E}+8$ & $\operatorname{cond}_{2}([[.,]])$. \\
\hline $2^{7}$ & $1.16 \mathrm{E}-12$ & $2.57 \mathrm{E}-12$ & $5.2 \mathrm{E}-10$ & $1.46 \mathrm{E}-4$ & $\left\|f-\Pi_{N}^{F}(f)\right\|_{\infty}$ \\
& $5.96 \mathrm{E}+3$ & $1.22 \mathrm{E}+6$ & $3.23 \mathrm{E}+7$ & $3.72 \mathrm{E}+13$ & $\operatorname{cond}_{2}([[.,]])$. \\
\hline $2^{8}$ & $5.61 \mathrm{E}-14$ & $1.86 \mathrm{E}-13$ & $1.9 \mathrm{E}-5$ & $6.6 \mathrm{E}-4$ & $\left\|f-\Pi_{N}^{F}(f)\right\|_{\infty}$ \\
& $2.5 \mathrm{E}+4$ & $8.08 \mathrm{E}+5$ & $3.09 \mathrm{E}+13$ & $1.1 \mathrm{E}+14$ & $\operatorname{cond}_{2}([[.,]])$. \\
\hline
\end{tabular}

TABLE 2. $\left\|f-\Pi_{N}^{F}(f)\right\|_{\infty}$ and $\operatorname{cond}_{2}([[.,]]$.$) as a function of the number of discretisation points$ $N$ and the parameter $\epsilon$, for $f(x)=\exp \left(-40(x-(2 \pi / 3))^{2}\right)$, with $h_{u}=2 \pi / N$. 

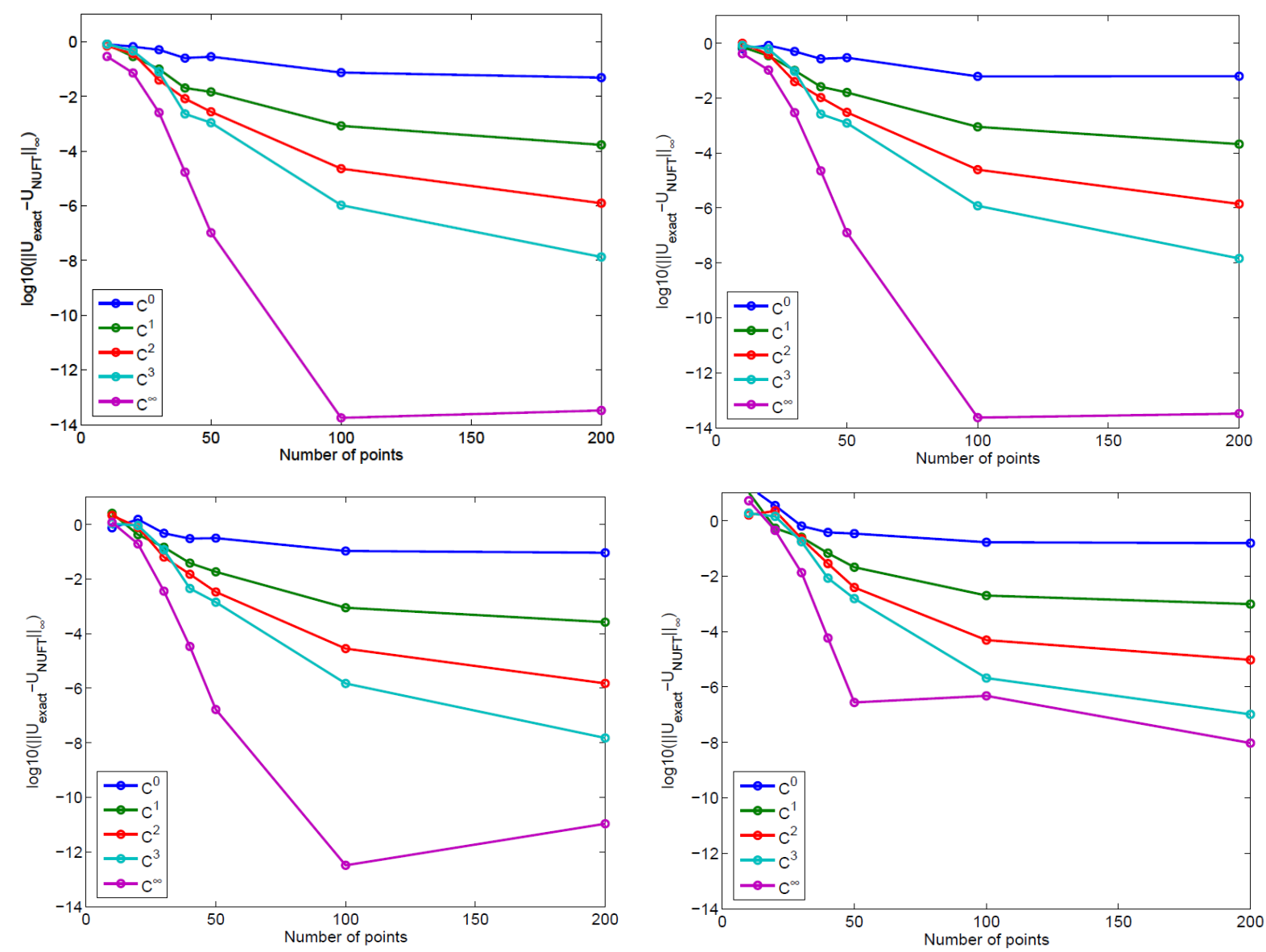

FIGURE 3. Approximation property for the 1D NUDFT with respect to the solution continuity with $\epsilon=h_{r e f} / 8, \epsilon=h_{r e f} / 4, \epsilon=h_{r e f} / 2, \epsilon=h_{r e f}$, from top left to bottom right.

\subsection{Numerical results for a nonseparable operator}

If the operator is nonseparable the operator $P$ is no longer diagonal.

Exemple 3.7. Let us consider the elliptic problem that follows:

$$
\begin{cases}-\nabla \cdot(a(x, y) \nabla u(x, y))=f(x, y) & \text { in } \Omega=[0, \pi]^{2}, \\ u=0 & \text { on } \partial \Omega\end{cases}
$$

with a DD in the $x$-direction into two overlapping subdomains. $a(x, y)=a_{0}+\left(1-a_{0}\right)\left(1+\tanh \left(\left(x-\left(3 * h_{x} *\right.\right.\right.\right.$ $\left.\left.\left.\left.y+1 / 2-h_{\text {ref }}\right)\right) / \epsilon_{a}\right)\right) / 2$, and $a_{0}=10, \epsilon_{a}=10^{-2}$, taking into account a jump not parallel to the interface between subdomains (see Figure 4 (left)). The right hand side $f$ is such that the exact solution is: $u(x, y)=$ $150 x(x-\pi) y(y-\pi)(y-\pi / 2) / \pi^{5}$. We use a Cartesian grid with $90 \times 90$ elements, uniform in $x$ and nonuniform in $y$ (the maximum distance between the grid points in the $y$-direction is of the order of $2 h_{\text {ref }},\left(\epsilon=h_{\text {ref }} / 2\right)$ ).

Figure 4 (right) represents Aitken-Schwarz convergence with respect to the Fourier modes kept to build the approximate $\tilde{P}_{[[., .]]}$for $N \in\{100,200,400\}$. It exhibits some continuity property with respect to the number of modes kept when $N$ increases. The first Fourier modes give a strong damping factor while the rest of Fourier 

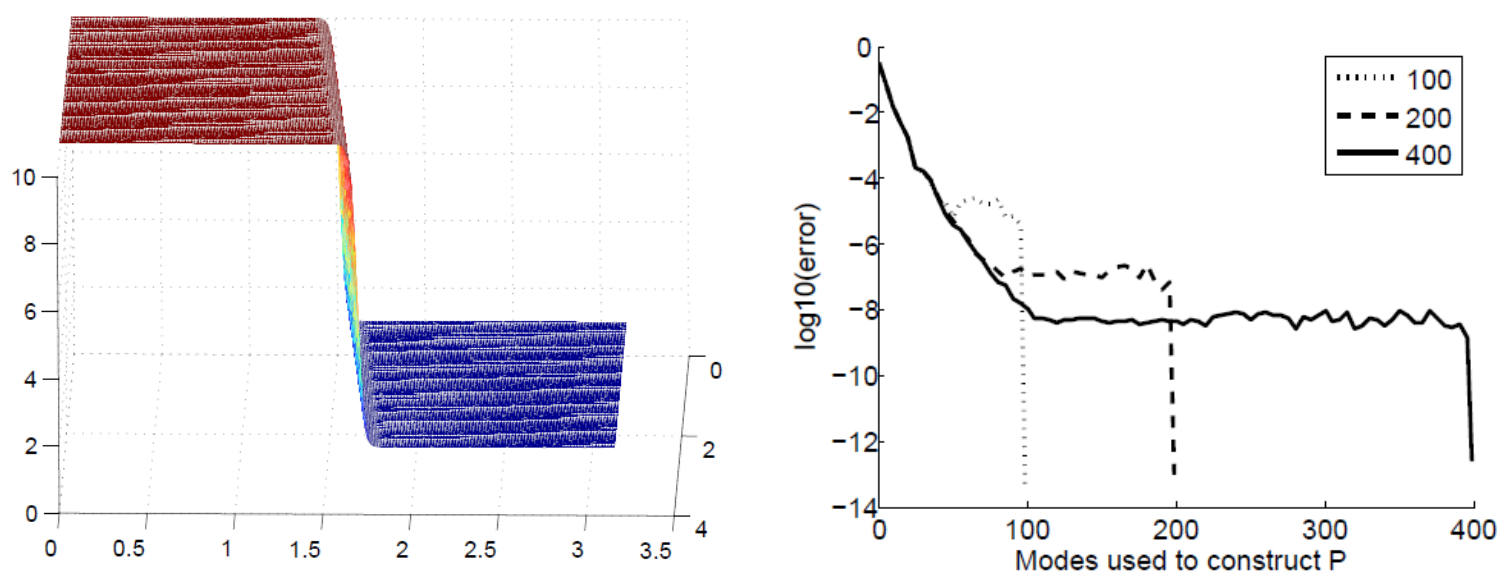

FiguRE 4 . The function $a(x, y)$ of the test problem, showing the jump not parallel to the interfaces (left). Influence of the approximation of $P_{[[., .]]}$on the interface error convergence for a strongly nonseparable operator on nonuniform grids (right).

modes does not improve so much the damping factor until reaching the full matrix where the total convergence damping is achieved due to algebraic considerations.

Figure 5 (left) exhibits the convergence of the Aitken-Schwarz method with respect to the number of modes kept to build the reduced matrix $\tilde{P}_{[[.,]]}$in accordance to algorithm (2).
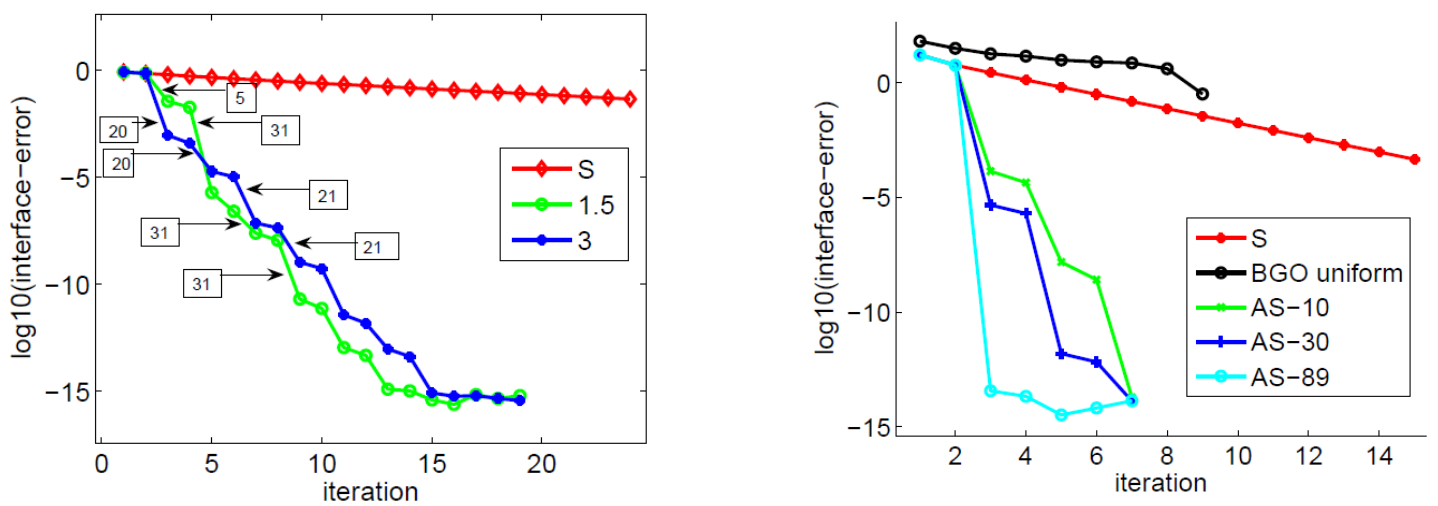

Figure 5. Adaptive acceleration using sub-blocks of $P_{[[. .,]]}$, with 100 points on the interface, overlap $=1, \epsilon=h_{\text {ref }} / 8$ and Fourier modes tolerance $=\left\|\hat{u}^{k}\right\|_{\infty} / 10^{i}$ for $i=1.5$ and 3 for 1 st iteration and $i=4$ for successive iterations (left). Acceleration using sub-blocks of $P_{[[., .]]}$with 90 points on the interface, overlap $=5$ and $\epsilon=h_{\text {ref }} / 2$. Top line refers to results in [1] (right).

On the other hand, in Figure 5 (right), the interface is nonuniform with $\epsilon=h_{r e f} / 2$. It shows good convergence properties even with 10 Fourier modes for the considered problem. The comparison with the result in [1] that considers a uniform mesh and an a priori five diagonal band approximation of the $P$ operator is assumed.

The techniques with Fourier transform need a sufficient number of points to approximate the interface in order to be able to express the solution in the base. In the following section we propose a different starting 
point to build the base. The main idea will be that we can compress the vectorial sequence using a Singular Value Decomposition (SVD). Since the matrix $\mathbb{U}$ is built, the matrix $P$ is built the same way.

\section{Approximation compressing the Vectorial Sequence}

A totally algebraic method based on the SVD of the Schwarz solutions on the interface has been proposed when the modes of the error could be strongly coupled [39]. This method offers the possibility for the Aitken Schwarz method to be used on a large class of problem without mesh consideration.

This section focuses on the definition of the orthogonal "base" $\mathbb{U}_{q}$. In the following, we use the term "base" to denote a set of spanning vectors that defines the approximation space. The key point of these preconditioners's efficiency is the choice of this orthogonal "base" $\mathbb{U}_{q}$. It must be sufficiently rich to numerically represent the solution at the interface, but it has to be not too large for the computation's efficiency.

Starting from the pure linear convergence $E^{j}=P E^{j-1}, \forall j \geq 1$ of the error $E^{j}$ of the Schwarz DD on the interface of size $n$ at the iteration $j$, one can formally build $P=\left[E^{n}, \ldots, E^{1}\right]\left[E^{n-1}, \ldots, E^{0}\right]^{-1}$. But the difficulty can be to invert the matrix $\left[E^{n-1}, \ldots, E^{0}\right]$ which can be close to singular. In a computation, most of the time is consumed solving some noise [40, chapter 8] that does not actually contribute to the solution. The SVD allows us to focus on the main part of the solution.

\subsubsection{The singular value decomposition}

A singular value decomposition (SVD) of a real $n \times m(n>m)$ matrix $A$ is its factorization into the product of three matrices:

$$
A=\mathbb{U} \Sigma \mathbb{V}^{*},
$$

where $\mathbb{U}=\left[U_{1}, \ldots, U_{m}\right]$ is a $n \times m$ matrix with orthonormal columns, $\Sigma$ is a $n \times m$ non-negative diagonal matrix with $\Sigma_{i i}=\sigma_{i}, 1 \leq i \leq m$ and the $m \times m$ matrix $\mathbb{V}=\left[V_{1}, \ldots, V_{m}\right]$ is orthogonal. The left $\mathbb{U}$ and right $\mathbb{V}$ singular vectors are the eigenvectors of $A A^{*}$ and $A^{*} A$ respectively. It readily follows that $A v_{i}=\sigma_{i} u_{i}, 1 \leq i \leq m$

We are going to recall some properties of the SVD. Assume that the $\sigma_{i}, 1 \leq i \leq m$ are ordered in decreasing order and there exists $r$ such that $\sigma_{r}>0$ while $\sigma_{r+1}=0$. Then $A$ can be decomposed in a dyadic decomposition:

$$
A=\sigma_{1} U_{1} V_{1}^{*}+\sigma_{2} U_{2} V_{2}^{*}+\ldots+\sigma_{r} U_{r} V_{r}^{*} .
$$

This means that SVD provides a way to find optimal lower dimensional approximations of a given series of data. More precisely, it produces an orthonormal base for representing the data series in a certain least squares optimal sense. This can be summarized by the theorem of Schmidt-Eckart-Young-Mirsky:

Théorème 4.1. A non unique minimizer $X_{*}$ of the problem $\min _{X, \text { rank } X=k}\|A-X\|_{2}=\sigma_{k+1}(A)$, provided that $\sigma_{k}>\sigma_{k+1}$, is obtained by truncating the dyadic decomposition of (56) to contain the first $k$ terms: $X_{*}=$ $\sigma_{1} U_{1} V_{1}^{*}+\sigma_{2} U_{2} V_{2}^{*}+\ldots+\sigma_{k} U_{k} V_{k}^{*}$

The SVD of a matrix is well conditioned with respect to perturbations of its entries. Consider the matrices $A, B \in \mathbb{R}^{n}$, the Fan inequalities write $\sigma_{r+s+1}(A+B) \leq \sigma_{r+1}(A)+\sigma_{s+1}(B)$ with $r, s \geq 0, r+s+1 \leq n$. Considering the perturbation matrix $E$ such that $\|E\|=O(\epsilon)$, then $\left|\sigma_{i}(A+E)-\sigma_{i}(A)\right| \leq \sigma_{1}(E)=\|E\|_{2}, \forall i$. This property does not hold for eigenvalues decomposition where small perturbations in the matrix entries can cause a large change in the eigenvalues.

This property allows us to search the acceleration of the convergence of the sequence of vectors in the base linked to its SVD.

Proposition 4.2. Let $\left(u^{k}\right)_{1 \leq k \leq q} q$ successive iterates satisfying the pure linear convergence property: $u^{k}-u^{\infty}=$ $P\left(u^{k-1}-u^{\infty}\right)$. Then there exists an orthogonal base $\mathbb{U}_{q}=\left[U^{1}, U^{2}, \ldots, U^{q}\right]$ of a subset of $\mathbb{R}^{n}$ such that $u^{k}=\sum_{l=1}^{q} \alpha_{l}^{k} U^{l}, \forall k \in\{1, \ldots, q\}$ with a decrease of $\alpha_{l}^{k}$ with respect to l for which one can write:

$$
\left(\alpha_{1}^{k+1}-\alpha_{1}^{k}, \ldots, \alpha_{q}^{k+1}-\alpha_{q}^{k}\right)^{t}=\hat{P}\left(\alpha_{1}^{k}-\alpha_{1}^{k-1}, \ldots, \alpha_{q}^{k}-\alpha_{q}^{k-1}\right)^{t}
$$


where $\hat{P} \stackrel{\text { def }}{=} \mathbb{U}_{q}^{*} P \mathbb{U}_{q}$. Moreover $\left(\alpha_{1}^{\infty}, \ldots, \alpha_{q}^{\infty}\right)^{t}$ obtained by the acceleration process represents the projection of the limit of the sequence of vectors in the space generated by $\mathbb{U}_{q}$.

Proof. By theorem 4.1 there exists a SVD decomposition of $\left[u^{1}, \ldots, u^{q}\right]=\mathbb{U}_{q} \Sigma^{*}$ and we can identify $\alpha_{l}^{k}$ as $\sigma_{l} V_{k l}^{*}$. The orthonormal property of $\mathbb{V}$ associated to the decrease of $\sigma_{l}$ with increasing $l$ leads to a decrease of $\alpha_{l}^{k}$ with respect to $l$. Taking the pure linear convergence of $u^{k}$ in the matrix form, and applying $\mathbb{U}_{q}$ leads to:

$$
\begin{array}{r}
\mathbb{U}_{q}^{*}\left(u^{k}-u^{\infty}\right)=\mathbb{U}_{q}^{*} P \mathbb{U}_{q} \mathbb{U}_{q}^{*}\left(u^{k-1}-u^{\infty}\right) \\
\left(\alpha_{1}^{k}-\gamma_{1}^{\infty}, \ldots, \alpha_{q}^{k}-\gamma_{q}^{\infty}\right)^{t}=\hat{P}\left(\alpha_{1}^{k-1}-\gamma_{1}^{\infty}, \ldots, \alpha_{q}^{k-1}-\gamma_{q}^{\infty}\right)^{t}
\end{array}
$$

where $\left(\gamma_{j}^{\infty}\right)_{1 \leq j \leq q}$ represents the projection of $u^{\infty}$ on the $\operatorname{span}\left\{U_{1}, \ldots, U_{q}\right\}$.

We can then derive Algorithm 3.

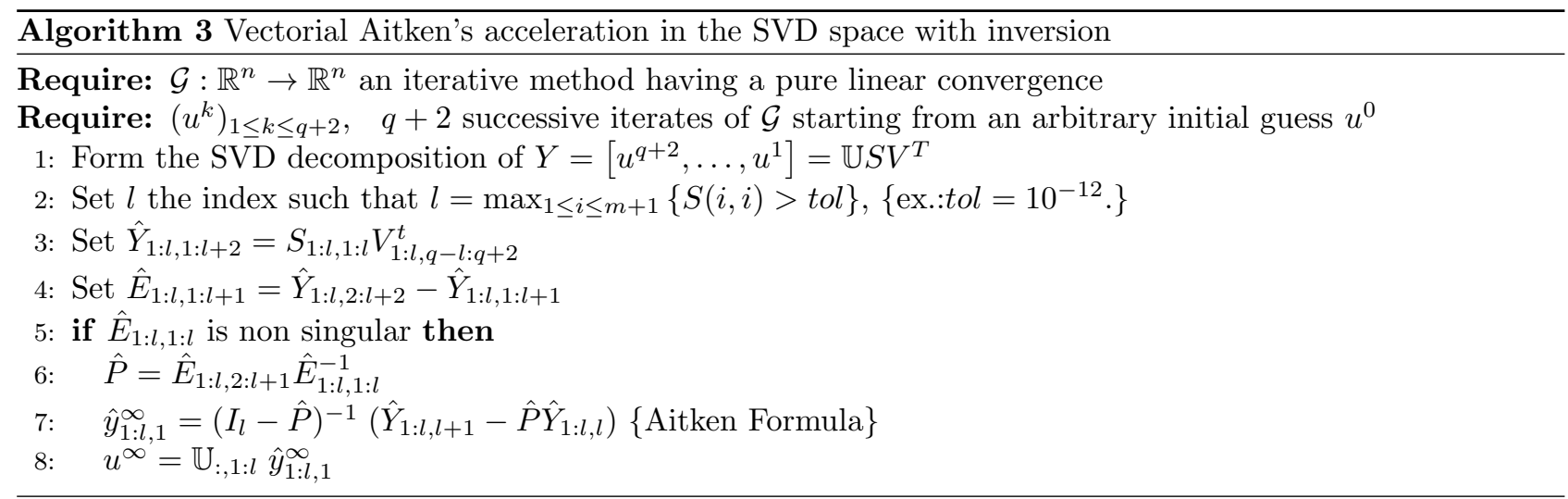

Proposition 4.3. Algorithm 3 converges to the limit $u^{\infty}$.

Proof. As the sequence of vector $u^{k}$ converges to a limit $u^{\infty}$ then we can write $\Xi=\left[u^{1}, \ldots, u^{q}\right]=\left[u^{\infty}, \ldots, u^{\infty}\right]+$ $E$ where $E$ is a $n \times q$ matrix with decreasing coefficients with respect to the columns. The SVD of $\Xi^{\infty}=$ $\left[u^{\infty}, \ldots, u^{\infty}\right]$ leads to have $U^{1}=u^{\infty}$ and $\sigma_{i}\left(\Xi^{\infty}\right)=0, i \geq 2$. The fan inequalities lead to have $\sigma_{i}(\Xi) \leq$ $\sigma_{1}(E)=\|E\|_{2}, i \geq 2$. Consequently, Algorithm 3 decreases the number of non zero singular values at each loop iterate.

In Algorithm 3 the building of $P$ needs the inversion of the matrix $\hat{E}_{1: l, 1: l}^{-1}$ which can contain very small singular values even if we selected those greater than a certain tolerance. This singular value can deteriorate the ability of $P$ to accelerate the convergence. If it is the case, we can proceed inverting this matrix with its SVD, replacing by zeros the singular values less than a tolerance instead of inverting them (see numerical recipes in [13]). A more robust algorithm can be obtained without inverting $\hat{E}_{1: l, 1: l}^{-1}$. It consists in building $P$ by applying the iterative method $\mathcal{G}$ to the selected columns of $\mathbb{U}_{q}$ that appears in Algorithm 3. Then $\hat{P}=\mathbb{U}_{1: n, 1: l}^{*} \mathcal{G}\left(\mathbb{U}_{1: n, 1: l}\right)$ as done in Algorithm 4.

\subsubsection{Computation of the Aitken's acceleration}

We now present two improvements of the Aitken's acceleration algorithm, we first give a criterion to choose the number of snapshots in order to get this burden removed from the user. Second, a generalization of the pseudoinverse by the truncation of small singular values of $\hat{E}_{n-1}$ is provided, allowing a more robust Aitken's acceleration.

In the previous algorithms, we assumed that the user provides a number of snapshots $n$ large enough to perform efficient accelerations. The acceleration can be inefficient and the solution may diverge when $n$ is 


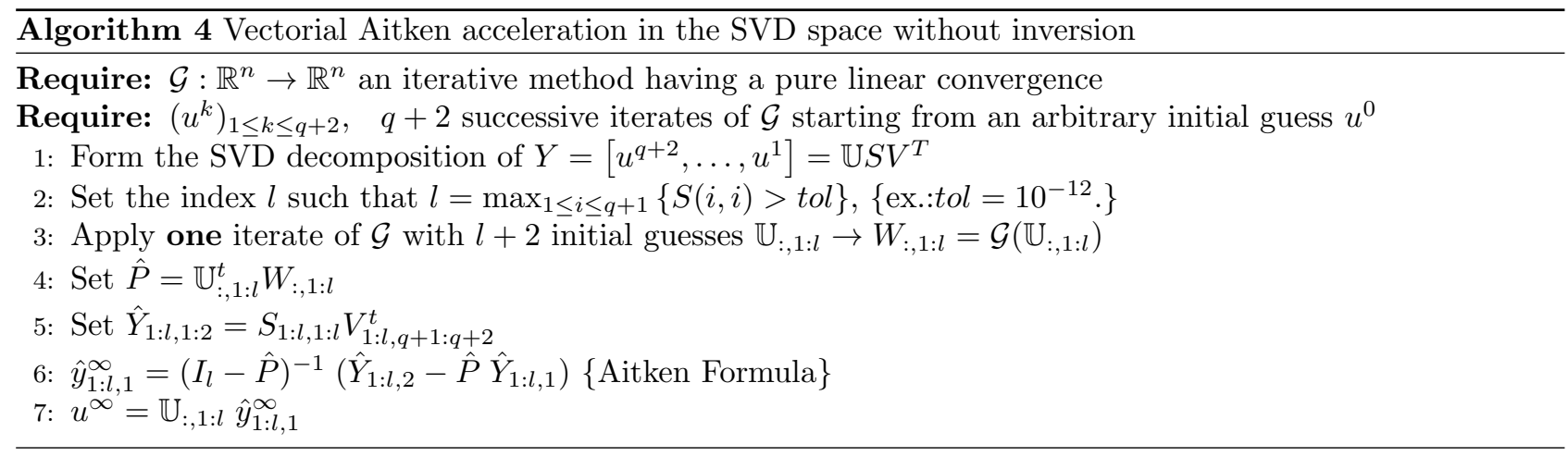

underestimated, but overestimating $n$ leads to perform useless iterations. To avoid overestimating $n$ while performing efficient acceleration we can compute the SVD on the fly during the Schwarz iterations. Thus the acceleration is performed when adding few snapshots does not lead to add columns in $\tilde{U}$. If $a$ is the number of additional snapshots and $S^{\prime}$ the new diagonal matrix containing singular values, then Schwarz iterations are stopped to perform the acceleration when Eq.(60) is satisfied.

$$
\max _{i=1 \ldots n}\left\{i, S_{i i}>S_{11} \times \epsilon_{F C G}\right\}=\max _{i=1 \ldots n+a}\left\{i, S_{i i}^{\prime}>S_{11}^{\prime} \times \epsilon_{F C G}\right\}
$$

To use this adaptive criterion we need to compute the SVD after each addition of snapshots. It can be recomputed from scratch or using the previous SVD thanks to an updating procedure [30, 38]. This updating procedure is called on-the-fly SVD. Since the computational time of the SVD is small relatively to Schwarz iterations, the key advantage of the updating procedure is to reduce the memory requirement: the SVD is truncated after each update, thus $\tilde{U}$ is computed from a $U \in \mathbb{R}^{m \times(l+a)}$ instead of a $U \in \mathbb{R}^{m \times n}$. For large problems $n$ is usually about twice greater than $l+a$, and $U$ is the largest matrix involved in the Aitken's acceleration, furthermore $U$ is computed on a single processor.

Even with a well computed $\tilde{U}$, the matrix $\hat{E}_{n-1}$ may have very small singular values associated to approximation errors. These small singular values become the largest ones of $\left(\hat{E}_{n-1}\right)^{-1}$ weakening the approximation of $\hat{P}$. Given the SVD $\hat{E}_{n-1}=V_{\hat{E}} S_{\hat{E}}^{\dagger} U_{\hat{E}}^{T}$, and a tolerance $\xi$, we can compute $\hat{P}$ as:

$$
\hat{P}=\hat{E}_{n} V_{\hat{E}} S_{\hat{E}}^{\ddagger} U_{\hat{E}}^{T} \text { with }\left(S_{\hat{E}}^{\ddagger}\right)_{i i}=\left\{\begin{array}{cll}
1 /\left(S_{\hat{E}}\right)_{i i} & \text { if } & \left(S_{\hat{E}}\right)_{i i} \geq \xi \\
0 & \text { if } & \left(S_{\hat{E}}\right)_{i i}<\xi
\end{array}\right.
$$

Unlike the SVD of the snapshots, we cannot use a truncation criterion based on the first singular value because the order of magnitude of this first singular value will decrease from an acceleration to another. Then, we set $\xi$ as the smallest singular value computed before the bend that we observe in the decrease of the singulars values.

\section{LARGe SCALE Numerical Results}

On the macroscopic scale, a porous medium can be described by a model where the solid and the fluid occupy the entire volume. The medium considered here is a parallelepiped domain modelled as a continuum where a representative volume element is larger than the average pore size. For this model of saturated flow in porous media, Darcy's law and mass conservation law give Eq.(62) where $u$ is the hydraulic head, and $K$ the permeability field.

$$
\left\{\begin{array}{c}
\nabla \cdot K \nabla u=q \text { in } \Omega \\
u=\alpha, \text { on } \Gamma_{1} \\
u=\beta, \text { on } \Gamma_{2} \\
\frac{\partial u}{\partial n}=0, \text { on } \partial \Omega \backslash\left(\Gamma_{1} \cup \Gamma_{2}\right)
\end{array}\right.
$$


The physical modelling of the heterogeneous media leads to several difficulties even for this linear Darcy equation. The treatment of various scales leads to solving sparse linear systems of very large size, with large condition number because of the heterogeneous permeability field $K$.

\subsection{Highly heterogeneous media}

Eq (62) is discretized using a regular grid on which a random permeability field $K$ is generated. This permeability field $K$ follows a stationary lognormal probability distribution $Y=\log (k)$, which is defined by a mean $m_{Y}$ set to zero, and a covariance function

$$
C_{Y}(x, y, z)=\sigma^{2} \exp \left(-\left[\left(\frac{x}{\lambda_{x}}\right)^{2}+\left(\frac{y}{\lambda_{y}}\right)^{2}+\left(\frac{z}{\lambda_{z}}\right)^{2}\right]^{\frac{1}{2}}\right)
$$

where $\sigma$ is the standard deviation of the $\log$ hydraulic conductivity and $\lambda_{x}, \lambda_{y}$ and $\lambda_{z}$ are the directional correlation lengths. To generate the random hydraulic field, a spectral simulation based on the Fast Fourier Transform method $[31,41]$ is used. For the sake of simplicity, we assume the media to be isotropic: $\lambda=\lambda_{x}=$ $\lambda_{y}=\lambda_{z}$. Then the covariance function becomes $C_{y}(r)=\sigma^{2}(-r / \lambda)$ where $r$ is the distance between two points.

Parameters $\sigma$ and $\lambda$ play a role in the stiffness of the linear system to be solved and in the convergence rate of the Schwarz method. Typically the condition number $\kappa(A)$ of the linear system is of the order of magnitude of $K_{\max } / K_{\min }$ where $K_{\min }$ and $K_{\max }$ are the minimal and the maximal values of the permeability. An example of a cut of three random permeability fields of $128^{3}$ points is given in Figure 6 to illustrate the effect of the parameters. The same random seed is used to generate these three fields.

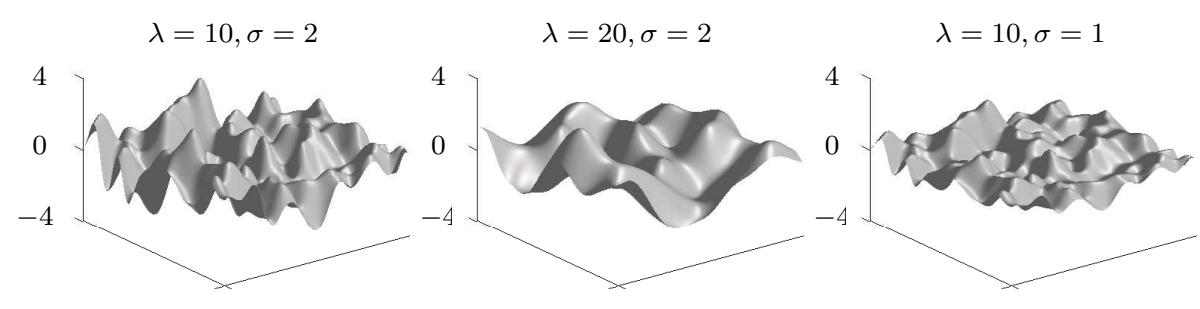

FIGURE 6. Values of the permeability in log10 scale for different parameters on a cut at $z=1$ of a permeability field of $128^{3}$ points. The $\lambda$ parameter plays on the smoothness of the variations, while the parameter $\sigma$ plays on the scale of the permeability values.

Let us consider a finite volume discretization of a domain $\Omega$ that leads to solve the linear system $A u=f$. Schwarz DD techniques allow us to solve the problem on $\Omega$ from the solutions on overlapping subdomains $\Omega_{i}$ such that $\Omega=\bigcup_{i=1}^{M} \Omega_{i}$. In the following we assume that a one-directional splitting of $\Omega$ is performed: each subdomain $\Omega_{i}$ has at most two neighboring subdomains $\Omega_{i-1}$ and $\Omega_{i+1}$. We define $A_{i} u_{i}^{k}=f_{i}^{k}$ as the local linear systems of the problem on $\Omega_{i}$ at the Schwarz iteration $k$. The local subdomain solver is the parallel Algebraical Mutigrid: AGMG of [29].

\subsection{Aitken-Schwarz as a specific solver for three-dimensional groundwater flow problems}

We now provide numerical tests for the purpose of answering to the questions of the robustness, of the choice between the two ways of compute $\hat{P}$, and of the scalability of the implementation. 


\subsubsection{Comparison of the approximations of $P$}

We are now interested in the comparison of both Aitken-Schwarz algorithms in terms of convergence and computational cost. Only the computation of $\hat{P}$ differs between the two algorithms: in algorithm $3 \hat{P}$ is computed as $\hat{P}=\hat{E}_{n}\left(\hat{E}_{n-1}\right)^{-1}$. On the one hand, the computational cost is very low since the dimension of the matrix $\left(\hat{E}_{n-1}\right)$ is very low. On the other hand, the inversion of $\left(\hat{E}_{n-1}\right)$ can be hazardous because the matrix $\hat{E}_{n-1}$ has been approximated. We consider a domain discretized in $1200 \times 420^{2} \approx 211 \times 10^{6}$ unknowns. The number of snapshots is not chosen adaptively, but fixed to ensure that the acceleration is performed after the same number of Schwarz iterations for both algorithms. This comparison is made for two permeability fields, the first one is generated according to the parameters $(\lambda, \sigma)=(10,1)$ and the second one according to $(\lambda, \sigma)=(20,3)$. Note that both parameters have been increased in order to slow the convergence rate of the Schwarz method. Convergences are plotted in Figure 7 . We remark that convergence are nearly the same for $(\lambda, \sigma)=(10,1)$. In that case algorithm 3 is more attractive since the computational is about $27 \mathrm{~min}$, instead of $33 \mathrm{~min}$ for algorithm 4. On the right hand side of Figure 7 we observe that algorithm 3 is less robust than algorithm 4 for stiff problems, but the computation of the pseudoinverse as in Eq.61 clearly improves the robustness.
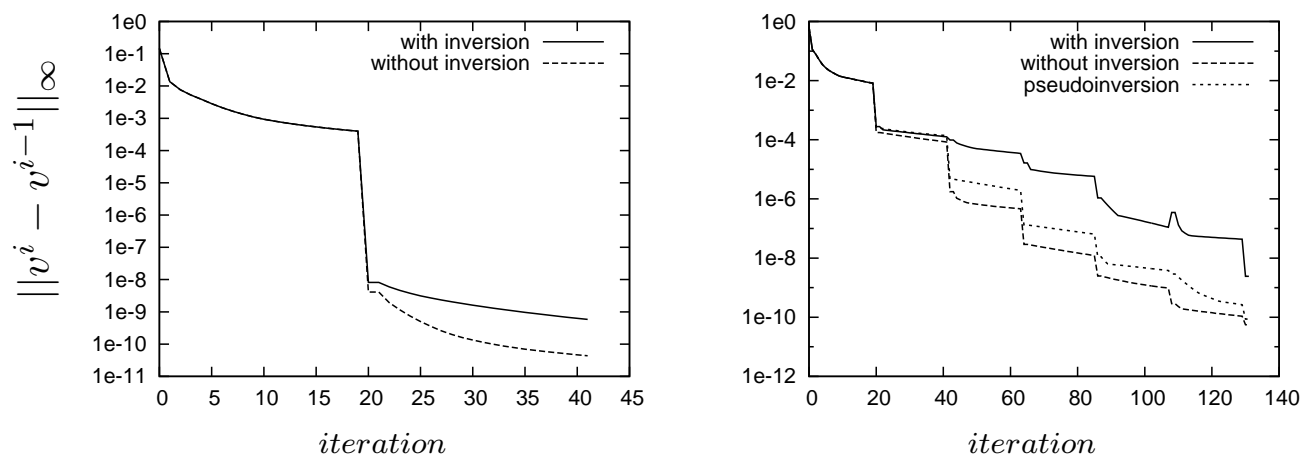

Figure 7. Error in log scale for $(\lambda, \sigma)=(10,1)$ (on the right) and $(\lambda, \sigma)=(20,3)$ (on the left)

\subsubsection{Weak Scaling test}

This implementation has been designed to provide a solution when it is not possible, or not desirable, to compute the solution on the whole problem. The number of subdomains should be as small as possible because the convergence rate of the Schwarz process, and the efficiency of the acceleration, decrease when the number of interfaces increases. Consequently, performing strong scaling tests would consist in increasing the number of processors per subdomain, which is equivalent to compute the strong scaling of the local solver.

To perform weak scaling tests without taking into account the weak scaling of the local solver, we fix the size of subdomains and the number of subdomains is increased. Algorithm 4 is used since it is the most robust, and the number of snapshots is chosen according to the adaptive criterion. Results are provided in Table 3 where:

- The number of iterations is the total number of Schwarz iterations (Algorithm 4 step 1) needed to reach the convergence.

- The walltime is the running time.

- The ratio is given by the total time per iteration.

The computational times of the SVD and the Aitken's formula grow with respect to the size of the problem, but they are still relatively small. These two steps are performed on a single processor but the size $U$ involved in 
TABLE 3. Weak scaling test: variation of the computational time when the size of the problem is increased, keeping the load per processor constant. The parameters are $(\lambda, \sigma)=(10,1)$

\begin{tabular}{ccccccc}
\hline \multirow{3}{*}{ Specifications } & Subdomains & 2 & 4 & 8 & 16 & 32 \\
& Processors & 128 & 256 & 512 & 1024 & 2048 \\
& Unknowns $\left(10^{6}\right)$ & 28.8 & 55.6 & 109 & 216 & 425 \\
\hline \multirow{3}{*}{ Scalability } & Number of iterations & 19 & 27 & 48 & 184 & 566 \\
& Walltime (min) & 4.6 & 5.3 & 9.6 & 36 & 102 \\
& Ratio & 0.242 & 0.196 & 0.200 & 0.195 & 0.180 \\
\hline
\end{tabular}

the SVD grows faster than the size of $\tilde{P}$ involved in the Aitken's formula. Performing the SVD itself in parallel could be needed for very large problems.

The number of iterations gives a numerical weak scaling. Notice that the complexity is less than quadratic which is acceptable for this kind of problems. Finally the ratio is nearly constant, so the weak scaling of our implementation well corresponds to the numerical weak scaling.

\section{Conclusions}

We showed the importance to represent cheaply the solution of the Schwarz DD method at the artificial interface generated by the domain decomposition. These solutions contain enough information in order to accelerate the convergence of the iterative method to the searched solution. The approximation space should be sufficiently rich to represent the searched solution. The nonuniform Fourier tansform allowed to develop an adaptative acceleration which gave us an estimate on the convergence for each Fourier-like mode. This allows us to select the modes that are involved in building the error operator $P$ on the interface. However, this nonuniform Fourier approximation is dependent on the regularity of the searched solution at the artificial interfaces. This is why the singular value decomposition of the iterated solutions provides a more general tool to build the approximation space. It still has the two major properties: an orthogonal "basis" to generate a vectorial subspace and a decreasing of the coefficients relative to this "basis" involved in the decomposition of the solution. This last property gives an estimate for the selection of the modes involved in the building of $P$. Then we succeed in performing large scale computing on problem of sizes that cannot be handled by the local (subdomain) solvers.

Some extensions of this methodology have been applied as preconditioning techniques for the Krylov solver. This is based on the acceleration of the Restrictive Additive Schwarz by Aitken with a Richardson formulation [10] [9]. Some illustrations of ill conditioned problems arising from computational fluid dynamics field can be found in [11].

This methodology can also be applied to time DD [23]. Some extensions to nonlinear problems are under consideration. In this case some use of the $\epsilon$-topological algorithm of [6] can replace the Aitken's acceleration of the convergence technique [21]. Another issue is to consider some nonlinear transmission conditions that makes the convergence of the Schwarz DD purely linear in a transformed space (with a nonlinear change of variable) $[22]$.

\section{AKNOWLEDGEMENT}

The author thanks one referee for pointing out many useful corrections for the readability of this paper.

\section{REFERENCES}

[1] J. Baranger, M. Garbey, and F. Oudin-Dardun. The Aitken-like acceleration of the Schwarz method on nonuniform Cartesian grids. SIAM J. Sci. Comput., 30(5):2566-2586, 2008. ISSN 1064-8275. . URL http://dx.doi.org/10.1137/050636607. 
[2] N Barberou, M Garbey, M Hess, MM Resch, T Rossi, J Toivanen, and D Tromeur-Dervout. Efficient metacomputing of elliptic linear and non-linear problems. JOURNAL OF PARALLEL AND DISTRIBUTED COMPUTING, 63(5):564-577, MAY 2003. ISSN 0743-7315. .

[3] L. Berenguer, T. Dufaud, and D. Tromeur-Dervout. Aitken's acceleration of the schwarz process using singular value decomposition for heterogeneous $3 d$ groundwater flow problems. Computers 8 Fluids, 80 (0):320-326, 2013. ISSN 0045-7930. . URL http://dx.doi.org/10.1016/j .compfluid.2012.01.026.

[4] John P. Boyd. Chebyshev and Fourier spectral methods. Dover Publications Inc., Mineola, NY, second edition, 2001. ISBN 0-486-41183-4.

[5] G. Bratu. Sur les équations intégrales non linéaires. Bull. Soc. Math. France, 42:113-142, 1914. ISSN 0037-9484.

[6] C. Brezinski. Convergence acceleration during the 20th century. J. Comput. Appl. Math., 122(1-2):1-21, 2000. ISSN 0377-0427. URL http://dx.doi.org/10.1016/S0377-0427 (00)00360-5. Numerical analysis 2000, Vol. II: Interpolation and extrapolation.

[7] MA Brune, GE Fagg, and MM Resch. Message-passing environments for metacomputing. FUTURE GENERATION COMPUTER SYSTEMS, 15(5-6):699-712, OCT 1999. ISSN 0167-739X.

[8] P. Chevalier and F. Nataf. An optimized order 2 (OO2) method for the Helmholtz equation. C. R. Acad. Sci. Paris Sér. I Math., 326(6):769-774, 1998. ISSN 0764-4442. . URL http://dx.doi.org/10.1016/S0764-4442(98)80047-5.

[9] T. Dufaud. Contribution to the development of Aitken Restricted Additive Schwarz preconditioning and application to linear systems arising from automatic differentiation of compressible Navier-Stokes solutions with respect to the simulation's parameters. PhD thesis, Université Lyon 1, 2011.

[10] Thomas Dufaud and Damien Tromeur-Dervout. Aitken's acceleration of the restricted additive Schwarz preconditioning using coarse approximations on the interface. C. R. Math. Acad. Sci. Paris, 348(13-14): 821-824, 2010. ISSN 1631-073X. . URL http://dx.doi.org/10.1016/j .crma.2010.06.021.

[11] Thomas Dufaud and Damien Tromeur-Dervout. Efficient parallel implementation of the fully algebraic multiplicative Aitken-RAS preconditioning technique. Advances in Engineering Software, 53:33-44, NOV 2012. .

[12] B. Engquist and H.-K. Zhao. Absorbing boundary conditions for domain decomposition. Appl. Numer. Math., 27(4):341-365, 1998. ISSN 0168-9274. . URL http://dx.doi.org/10.1016/S0168-9274(98)00019-1.

[13] B. Flannery, W. H. Press, S. Teukolsky, and W. Vetterling, editors. Numerical Recipes: The Art of Scientific Computing. Cambridge University Press, third edition, 2007.

[14] A. Frullone and D. Tromeur-Dervout. A new formulation of nudft applied to aitken-schwarz ddm on nonuniform meshes. In Parallel Computational Fluid Dynamics 2005, pages 493-500, 2006.

[15] M. J. Gander, F. Magoulès, and F. Nataf. Optimized Schwarz methods without overlap for the Helmholtz equation. SIAM J. Sci. Comput., 24(1):38-60 (electronic), 2002. ISSN 1064-8275. . URL http://dx.doi.org/10.1137/S1064827501387012.

[16] M. Garbey. Acceleration of the Schwarz method for elliptic problems. SIAM J. Sci. Comput., 26(6): 1871-1893, 2005. ISSN 1064-8275. . URL http://dx.doi.org/10.1137/S1064827502416344.

[17] M. Garbey and D. Tromeur-Dervout. Two level domain decomposition for multiclusters. In T. Chan \& Al editors, editor, 12th Int. Conf. on Domain Decomposition Methods DD12, pages 325-339. ddm.org, 2001.

[18] M. Garbey and D. Tromeur-Dervout. On some Aitken-like acceleration of the Schwarz method. Internat. J. Numer. Methods Fluids, 40(12):1493-1513, 2002. ISSN 0271-2091. . URL http://dx.doi.org/10.1002/fld.407. LMS Workshop on Domain Decomposition Methods in Fluid Mechanics (London, 2001).

[19] L. Gerardo-Giorda and F. Nataf. Optimized Schwarz methods for unsymmetric layered problems with strongly discontinuous and anisotropic coefficients. J. Numer. Math., 13(4):265-294, 2005. ISSN 15702820. . URL http://dx.doi.org/10.1163/156939505775248338.

[20] Peter Henrici. Elements of numerical analysis. John Wiley \& Sons Inc., New York, 1964. 
[21] P. Linel. Méthodes de décomposition de domaines en temps et en espace pour la résolution de systèmes d'EDO non-linéaires. PhD thesis, Université Lyon 1, 2011.

[22] P. Linel and D. Tromeur-Dervout. Nonlinear transmission conditions for time domain decomposition method. In Proc. DD21, submitted, 2013.

[23] Patrice Linel and Damien Tromeur-Dervout. Une méthode de décomposition en temps avec des schémas d'intégration réversible pour la résolution de systèmes d'équations différentielles ordinaires. C. R. Math. Acad. Sci. Paris, 349(15-16):911-914, 2011. ISSN 1631-073X. . URL http://dx.doi.org/10.1016/j.crma.2011.07.002.

[24] P.-L. Lions. On the Schwarz alternating method. I. In First International Symposium on Domain Decomposition Methods for Partial Differential Equations (Paris, 1987), pages 1-42. SIAM, Philadelphia, PA, 1988.

[25] P.-L. Lions. On the Schwarz alternating method. III. A variant for nonoverlapping subdomains. In Third International Symposium on Domain Decomposition Methods for Partial Differential Equations (Houston, TX, 1989), pages 202-223. SIAM, Philadelphia, PA, 1990.

[26] L. D. Marini and A. Quarteroni. A relaxation procedure for domain decomposition methods using finite elements. Numer. Math., 55(5):575-598, 1989. ISSN 0029-599X. . URL http://dx.doi.org/10.1007/BF01398917.

[27] Ramesh Natarajan. Domain decomposition using spectral expansions of Steklov-Poincaré operators. II. A matrix formulation. SIAM J. Sci. Comput., 18(4):1187-1199, 1997. ISSN 1064-8275. . URL http://dx.doi.org/10.1137/S1064827594274309.

[28] Jindřich Nečas. Sur une méthode pour résoudre les équations aux dérivées partielles du type elliptique, voisine de la variationnelle. Ann. Scuola Norm. Sup. Pisa (3), 16:305-326, 1962.

[29] Y. Notay. An aggregation-based algebraic multigrid method. Electronic Transactions on Numerical Analysis, 37, 2010.

[30] R. Martin P. Hall, D. Marshall. Adding and subtracting eigenspaces with eigenvalue decomposition and singular value decomposition. Image and Vision Computing, 20(13-14):1009-1016, 2002.

[31] E. Pardo-Igúzquiza and M. Chica-Olmo. The fourier integral method: an efficient spectral method for simulation of random fields. Mathematical Geology, 25:177-216, 1993.

[32] A. Quarteroni and A. Valli. Domain decomposition methods for partial differential equations. Numerical Mathematics and Scientific Computation. The Clarendon Press Oxford University Press, New York, 1999. ISBN 0-19-850178-1. Oxford Science Publications.

[33] Tuomo Rossi and Jari Toivanen. A nonstandard cyclic reduction method, its variants and stability. SIAM J. Matrix Anal. Appl., 20(3):628-645 (electronic), 1999. ISSN 0895-4798. . URL http://dx.doi.org/10.1137/S0895479897317053.

[34] Tuomo Rossi and Jari Toivanen. A parallel fast direct solver for block tridiagonal systems with separable matrices of arbitrary dimension. SIAM J. Sci. Comput., 20(5):1778-1796, 1999. ISSN 1064-8275. . URL http://dx.doi.org/10.1137/S1064827597317016.

[35] H. A. Schwarz. Vierteljahresschrift der naturforschenden gesellschaft. Zurich, 15:272-286, 1870.

[36] A. St-Cyr, M. J. Gander, and S. J. Thomas. Optimized multiplicative, additive, and restricted additive Schwarz preconditioning. SIAM J. Sci. Comput., 29(6):2402-2425 (electronic), 2007. ISSN 1064-8275. . URL http://dx.doi.org/10.1137/060652610.

[37] Olaf Steinbach. Stability estimates for hybrid coupled domain decomposition methods, volume 1809 of Lecture Notes in Mathematics. Springer-Verlag, Berlin, 2003. ISBN 3-540-00277-4. . URL http://dx.doi.org/10.1007/b80164.

[38] D. Tromeur-Dervout T. Pham. Proper orthogonal decomposition in decoupling dynamical systems. Proceedings of the Second International Conference on Parallel, Distributed, Grid and Cloud Computing for Engineering, Civil-Comp Press, Stirlingshire, UK, Paper 55, 2011. 
[39] D. Tromeur-Dervout. Meshfree Adaptative Aitken-Schwarz Domain Decomposition with application to Darcy Flow. In Topping, BHV and Ivanyi, P, editor, Parallel, Distributed and Grid Computing for Engineering, volume 21 of Computational Science Engineering and Technology Series, pages 217-250. SaxeCoburg Publications, Stirlingshire, UK, 2009. URL http://dx.doi.org/10.4203/csets.21.11.

[40] Henk A. van der Vorst. Iterative Krylov methods for large linear systems, volume 13 of Cambridge Monographs on Applied and Computational Mathematics. Cambridge University Press, Cambridge, 2009. ISBN 978-0-521-18370-3. Reprint of the 2003 original.

[41] Yao. Reproduction of the mean, variance, and variogram model in spectral simulation. Mathematical Geology, 36:487-506, 2004. 